\title{
The sphericity of central heavy-ion reactions
}

\author{
W. J. Llope, ${ }^{*}$ W. Bauer, ${ }^{2}$ D. Craig, ${ }^{2}$ E. E. Gualtieri, ${ }^{2}$ S. Hannuschke, ${ }^{2}$ R. A. Lacey, ${ }^{3}$ J. Lauret, ${ }^{3}$ T. Li, ${ }^{2}$ C. M. Mader, ${ }^{8}$ \\ A. Nadasen, ${ }^{4}$ E. Norbeck, ${ }^{7}$ R. Pak, ${ }^{2}$ G. Peilert, ${ }^{6}$ N. T. B. Stone, ${ }^{2}$ A. M. Vander Molen, ${ }^{2}$ G. D. Westfall, ${ }^{2}$ J. Yee, ${ }^{2}$ \\ and S. J. Yennello ${ }^{5}$ \\ (The NSCL $4 \pi$ Group) \\ ${ }^{1}$ T. W. Bonner Nuclear Laboratory, Rice University, Houston, Texas 77251 \\ ${ }^{2}$ National Superconducting Cyclotron Laboratory, Michigan State University, East Lansing, Michigan 48824 \\ ${ }^{3}$ Department of Chemistry, State University of New York - Stony Brook, Stony Brook, New York 11794 \\ ${ }^{4}$ Department of Physics, University of Michigan - Dearborn, Dearborn, Michigan 48128 \\ ${ }^{5}$ Cyclotron Institute, Texas A\&M University, College Station, Texas 77843 \\ ${ }^{6}$ Lawrence Livermore National Laboratory, Livermore, California 94550 \\ ${ }^{7}$ Department of Physics and Astronomy, University of Iowa, lowa City, Lowa 52242 \\ ${ }^{8}$ Department of Physics, Hope College, Holland, Michigan 49423
}

(Received 22 December 1994)

\begin{abstract}
We have experimentally studied small impact parameter heavy-ion collisions in the (nearly) symmetry entrance channels ${ }^{12} \mathrm{C}+{ }^{12} \mathrm{C},{ }^{20} \mathrm{Ne}+{ }^{27} \mathrm{Al},{ }^{40} \mathrm{Ar}+{ }^{45} \mathrm{Sc},{ }^{84} \mathrm{Kr}+{ }^{93} \mathrm{Nb}$, and ${ }^{129} \mathrm{Xe}+{ }^{139} \mathrm{La}$, each at many intermediate beam energies. The results from a number of analyses based on a projection of the "shapes" of the experimental events called the sphericity are presented. Comparisons of the relative efficiencies of various experimental methods for the selection of central events are made. The importance of autocorrelations between the sphericity and the various impact-parameter-dependent variables is evaluated. Searches for beam energydependent transitions from sequential binary disassembly to multifragmentation in the central events are described. Comparisons to dynamic and hybrid model code calculations will be discussed. The average sphericities of the intermediate mass fragments (IMF's, for which $3 \leqslant Z \leqslant 20$ ), are presented. The possibility that the IMF emission occurs following the formation of transient toroidal or disk-like geometries in the central events is explored. Increases in the average sphericities of the central events for increasing beam energies are observed which is attributed to transitions from sequential binary disassembly to multifragmentation. The transitional beam energies for the central ${ }^{40} \mathrm{Ar}+{ }^{45} \mathrm{Sc},{ }^{84} \mathrm{Kr}+{ }^{93} \mathrm{Nb}$, and ${ }^{129} \mathrm{Xe}+{ }^{139} \mathrm{La}$ reactions are near $\sim 50$, $\sim 40$, and $\sim 40 \mathrm{MeV} /$ nucleon, respectively.
\end{abstract}

PACS number(s): 25.70.Pq, 25.70.-z, 25.70.Jj, 25.70.Lm

\section{INTRODUCTION}

It is possible to form excited nuclear systems in the laboratory by colliding atomic nuclei. The impact parameter, as well as the predominant reaction mechanisms at each impact parameter, can be inferred from the experimentally measured characteristics of the particle emission. Given an efficient experimental selection of the most central collisions, beam energies from $\sim 15$ to $\sim 150 \mathrm{MeV} /$ nucleon can result in the formation of nuclear systems with excitation energies from several to tens of $\mathrm{MeV} /$ nucleon. In such a range of excitation energies, previous experiments have indicated possible transitions between sequential binary (SB) disassembly mechanisms and multifragmentation (MF) (see Ref. [1] for recent reviews). Detailed theoretical calculations [2-4] have predicted that the equivalent of a proper liquid-gas phase transition in finite nuclear systems occurs at excitation energies on the order of $10 \mathrm{MeV} /$ nucleon for systems of mass $\sim 100$. The possibility that transitions in disassembly mode from $\mathrm{SB}$ to $\mathrm{MF}$ are an artifact of such a liquid-gas phase transition is, however, only one of many. Systematic experimental studies of the total mass and excitation energy dependence of the predominant disassembly mechanisms and the

"Electronic address: Hope@physics.rice.edu applicability of the various theoretical descriptions are therefore necessary. In this paper, such studies based on event shape analyses of a comprehensive set of experimental data are described.

The experimental data were collected using the MSU $4 \pi$ Array [5] at the National Superconducting Cyclotron Laboratory. Reactions in the entrance channels ${ }^{12} \mathrm{C}+{ }^{12} \mathrm{C}$, ${ }^{20} \mathrm{Ne}+{ }^{27} \mathrm{Al},{ }^{40} \mathrm{Ar}+{ }^{45} \mathrm{Sc},{ }^{84} \mathrm{Kr}+{ }^{93} \mathrm{Nb}$, and ${ }^{129} \mathrm{Xe}+{ }^{139} \mathrm{La}$ were measured $[6,7]$ with a minimum bias trigger in $5-10 \mathrm{MeV} /$ nucleon steps in beam energy, up to the maximum energy available from the $\mathrm{K} 1200$ Cyclotron for each projectile: 155 , $140,115,75$, and $60 \mathrm{MeV} /$ nucleon, respectively. The lowest beam energies measured in each entrance channel were 55 , $55,15,35$, and $25 \mathrm{MeV} /$ nucleon, respectively. The apparatus consists of a main ball of 170 phoswich detectors in a closepacked geometry covering the laboratory polar angles from $\sim 16^{\circ}$ to $\sim 164^{\circ}$, and a 45 -element phoswich forward array covering from $\sim 4^{\circ}$ to $\sim 16^{\circ}$. The phoswich detectors of the main ball are fronted by 55 Bragg curve counters (BCC's), which were run in ion-chamber mode. The kinetic energy thresholds are typically $\sim 17$ (5) MeV/nucleon for protons $\left({ }^{12} \mathrm{C}\right.$ fragments). The main ball phoswich detectors resolve charges from $Z=1$ to $\sim 8$, while the BCC's resolve $Z=2$ to $\sim 18$, and the forward array resolves $Z=1$ to $\sim 15$. All of the phoswich detectors provide isotopic resolution for $Z=1$ particles. The phoswich detectors are quite thick; protons with 


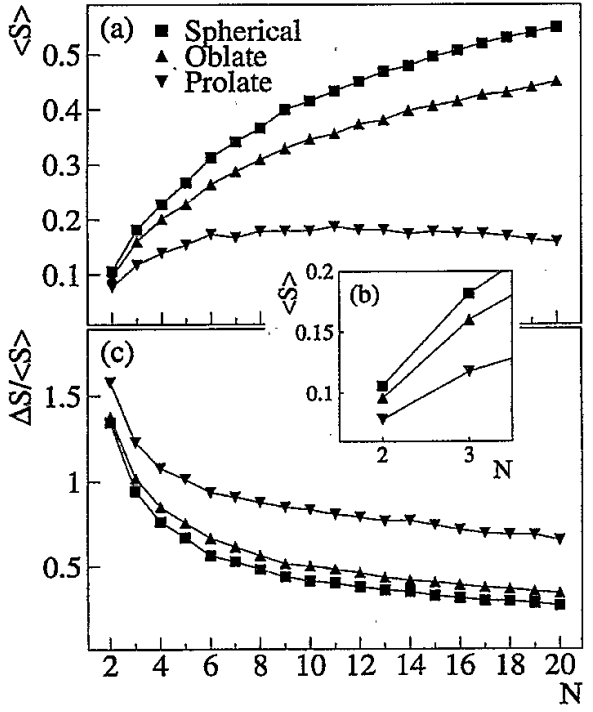

FIG. 1. The average sphericity [(a) and (b)], and the sphericity width divided by the average (c), versus the particle multiplicity for spherical (squares), oblate (up triangles), and prolate (down triangles) particle emission patterns generated as described in Ref. [11].

energies $\leqslant 250 \mathrm{MeV}$, and all of the fragments, do not punch through.

The analyses of these data will proceed via the study of event shapes. These summarize particular aspects of the three-dimensional average patterns of the particle emission in a momentum space coordinate system that spatially coincides with the center of momentum (CM) frame. The Cartesian components of the $\mathrm{CM}$ frame particle momenta, $p^{(k)}$, are used to fill the "flow tensor" [8] $F_{i j}=\Sigma_{k}^{N}\left[p_{i}^{(k)} p_{j}^{(k)} /\right.$ $\left.2 m_{k}\right]$ in each event. The normalization of the eigenvalues of this tensor $t_{i}$ via $q_{i}=t_{i}^{2} / \Sigma_{i=1}^{3} t_{i}^{2}$ allows the calculation of the sphericity [9] using $S=\frac{3}{2}\left(1-q_{3}\right)$, where $q_{3}$ is the largest normalized eigenvalue. All shape observables extracted from $F_{i j}$, e.g., $S$, depend strongly $[10,11]$ on the number of particles, $N$, included in the sum in $F_{i j}$. For a given value of $N$, particle emission patterns that are isotropic in the momentum space coordinate system spatially coinciding with the CM frame have the largest possible sphericities, while otherwise deformed emission patterns have smaller sphericities.

The finite multiplicity distortions affect both the average values and the widths of all shape observables. This is shown in Fig. 1, where the average sphericity $\langle S\rangle$ and reduced sphericity width $\Delta S /\langle S\rangle=\sqrt{\left\langle S^{2}\right\rangle-\langle S\rangle^{2}} /\langle S\rangle$ are plotted versus $N$ for spherical, prolate, and oblate particle emission patterns. For each multiplicity, $\sim 50000$ events are generated for each shape as described in Ref. [11]. The strong dependence of $\langle S\rangle$ and $\Delta S /\langle S\rangle$ on the number of particles in the event is apparent in Figs. 1(a) and 1(c). Distinct differences in $\langle S\rangle$ are observed for all multiplicities [cf. Figs. 1(a) and 1(b)]. Even at a multiplicity of 2 , the average sphericity of spherical emission patterns exceeds that for prolate (oblate) patterns by $\sim 0.03$ (0.01). Larger multiplicities lead to larger differences between the average sphericities of the different shapes. While the sphericity widths increase with decreasing multiplicities, accurate measurements of the average values are possible given sufficient statistics. For all of the analyses of experimental data described in the following sections, more than $\sim 2.5 \times 10^{6}$ minimum bias, and $\sim 0.1 \times 10^{6}$ central events, were studied. This allows one to distinguish spherical emission patterns from prolate or oblate ones, even in instances where the particle multiplicity, and hence the differences between the average sphericities of the different shapes, is small.

Given methods to remove the dependence of $\langle S\rangle$ on $N$, it is possible to extract information concerning the impact parameter and the characteristics of the predominant reaction mechanisms from this observable. The sensitivity of the sphericity to the impact parameter is caused by the increasing probability for the emission of particles from spectatorlike sources as the impact parameter is increased [12]. Particles emitted from such sources have relatively large momenta and forward/backward focused emission angles when viewed from the CM frame. Large impact parameter events thus lead to prolate particle emission patterns, which have strongly suppressed average sphericities. Also, in the most central collisions, SB disassembly of the excited system results in emission patterns that are more elongated in momentum space than those expected for MF decays $[9,13]$. In a region of excitation energy and mass for which the first decay step involves an (a)symmetric fission, the particles in the final state kinematically reflect the back-to-back trajectories of the two excited pre-fragments produced following the fission step.

The finite values of the multiplicities, $N$, and inefficiencies in the central event selection conspire to suppress $\langle S\rangle$, and to decrease the distinctions in average sphericity between SB and MF events. Concerning the former, we will therefore only present results following the placement of some constraint on the multiplicity of particles used in the calculation of the sphericity for each event. To limit the latter, we begin by investigating the relative efficiencies of various experimental methods for selecting the central collisions, which is described in Sec. I. We also present evidence for a beam-energy-dependent change in the predominant decay mechanism in the central events in this section. Additional evidence for such transitions is described in Secs. II and IIII. Section II describes comparisons to the average sphericities of the filtered events from a number of SB and MF models, while Sec. III presents "subset sphericities." The possibility of the formation of transient toroidal or disk-shaped freezeout configurations in the central events is also explored in Sec. III. The summary and conclusions are presented in Sec. IV.

\section{THE SELECTION OF CENTRAL COLLISIONS}

The selection of the most central experimental collisions constrains the mass of the excited nuclear system and results in a monotonic relationship between the beam energy and the excitation energy in this system. Software cuts on global observables, i.e., centrality variables, that are assumed to be correlated with the impact parameter are used to select samples of the most central events. One must ensure, however, that the specific cut used to select these events is relatively inefficient at selecting larger impact parameter events with significant topological fluctuations, and does not autocorrelate with subsequent stages of the analysis. A discussion. 
of these two issues is presented in this section.

Geometrical arguments, e.g., from Ref. [14], allow one to relate the fraction of minimum bias events selected by a particular centrality cut to an average impact parameter leading to these events. Such arguments assume only that a given centrality variable is correlated with the impact parameter, but do not consider that different centrality variables may be more or less tightly correlated with this parameter. Comparisons of the degree of correlation between different centrality variables and the impact parameter in heavy-ion collisions have previously been performed by several groups, each using different techniques. Tsang et al. [15] used the linear momentum transfer via the fission fragment fold angles to investigate the sensitivity on the impact parameter of various angular cuts on the charged particle multiplicity for ${ }^{36} \mathrm{Ar}$ and ${ }^{14} \mathrm{~N}$ induced reactions on ${ }^{238} \mathrm{U}$ targets. Ogilvie and collaborators [16] used simulations to investigate the correlations between several centrality variables and the magnitude and direction of the impact parameter vector for symmetric collisions of mass -40 nuclei. Also, Phair et al. [17,18] used three-source fits, the suppression of projectilelike fragments, and azimuthal correlations to investigate the sensitivity of several different centrality variables for ${ }^{36} \mathrm{Ar}+{ }^{197} \mathrm{Au}$ collisions. In this section, we present a study of the degree of correlation between six different centrality variables and the magnitude of the impact parameter. The dependence of the relative strengths of these correlations on the beam energy and entrance channel mass in symmetric collisions will be presented. The present study is based on the information provided by the average sphericity of the events.

In peripheral collisions, the kinetic energy and polar angle dependence of particle emission can be approximately parametrized as that from two independent thermal sources which move through the CM frame with projectilelike and targetlike velocities. In such a picture, increasingly more central collisions result in larger cross sections for particle emission from a source at rest in the $\mathrm{CM}$ frame. The relative velocities of the spectator source frames and the CM frame lead to the appearance of large kinetic energies and strong forward-backward focusing of the particles emitted from spectator sources, when such emission is viewed from the CM frame. Particle emission from spectatorlike sources thus elongates the shape of the event in the CM frame, and leads to severe suppressions of the sphericity that are related directly to the impact parameter.

That the average sphericity is indicative of the impact parameter assuming a perfect experimental apparatus, i.e., for unfiltered model calculations, is shown in, e.g., Ref. [12]. Before one can rank the sensitivity of various centrality variables to the impact parameter for experimental data, one must first prove that the average sphericity is still sensitive to the impact parameter including any experimental inefficiencies present during the data collection. We have investigated this question for the present data using simulations that include a detailed software replica of the MSU $4 \pi$ Array. The simulations and the filter code used to reproduce all of the experimental inefficiencies are described in more detail in Sec. II below and in Ref. [7].

Typical results are shown in Fig. 2, which depicts the average sphericity versus the impact parameter obtained from filtered Quantum Molecular Dynamics (QMD) +

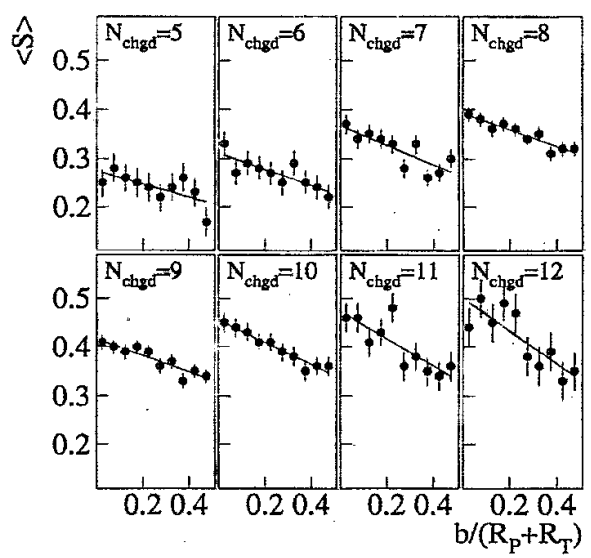

FIG. 2. The average sphericity versus the reduced impact parameter at specific multiplicities for the QMD+ COPENHAGEN simulations of ${ }^{40} \mathrm{Ar}+{ }^{45} \mathrm{Sc}$ collisions at a beam energy of $45 \mathrm{MeV} /$ nucleon. The solid lines are linear fits included only to guide the eye. The generated events were filtered using the detailed software replica of the MSU $4 \pi$ Array, which includes all of the experimental inefficiencies.

COPENHAGEN (see below) simulations of ${ }^{40} \mathrm{Ar}+{ }^{45} \mathrm{Sc}$ collisions at a beam energy of $45 \mathrm{MeV} /$ nucleon. The solid lines are linear fits included only to guide the eye. A soft equation of state $\left(K_{\infty}=200 \mathrm{MeV}\right)$ and a flat impact parameter distribution from zero to $4 \mathrm{fm}\left[b \leqslant\left[R_{P}+R_{T}\right] / 2\right.$ for this reaction, where $R_{P}\left(R_{T}\right)$ is the radius of the projectile (target) nucleus] were used in the QMD calculation. The freeze-out density used in the COPENHAGEN calculation was one-third of normal nuclear matter density.

According to Fig. 2, the values of $\langle S\rangle$ in the filtered events are maximal at impact parameters near zero, and decrease significantly as the impact parameter is increased. This sensitivity of $\langle S\rangle$ on the impact parameter in the filtered events is apparent for all values of the multiplicity. Similar results are obtained for other beam energies in the filtered $\mathrm{QMD}+\mathrm{COPENHAGEN} \mathrm{simulations} \mathrm{of} \mathrm{the}{ }^{40} \mathrm{Ar}+{ }^{45} \mathrm{Sc}$ reactions, and for the other entrance channels, which were studied using filtered FREESCO [9] simulations.

The experimental selection of the most central collisions proceeds by the selection of those events with specific values of a global observable that is assumed to be monotonic with the impact parameter $[16,17]$. Examples of such centrality variables are the measured total charged particle multiplicity $N_{\text {chgd }}$, the total charge detected in a software gate centered at midrapidity, $Z_{\mathrm{MR}}$, the proton multiplicity $N_{p}$ (which is complementary to the total charge bound in fragments, $\left.Z_{\text {bound }}\right)$, the total detected charge $Z_{\text {det }}$, and the total transverse kinetic energy $E_{T}$. In the present study, all of these variables are defined as described in Refs. [16] and [17], with the exception of the variables complementary to $Z_{\text {bound }}$. The proton multiplicity $N_{p}$ is used herein as the complement to the total charge in fragments with $A \geqslant 2$ (previously, the requirement $Z \geqslant 2$ was used, and the complement was the hydrogen multiplicity). For each of these observables, maximal values are assumed to occur in the smallest impact parameter collisions. From (approximate) geometrical arguments [14], the selection of the $\sim 10 \%$ most central events results in average impact parameters of $\langle b\rangle \sim 0.3 b_{\max }$. The quantity 


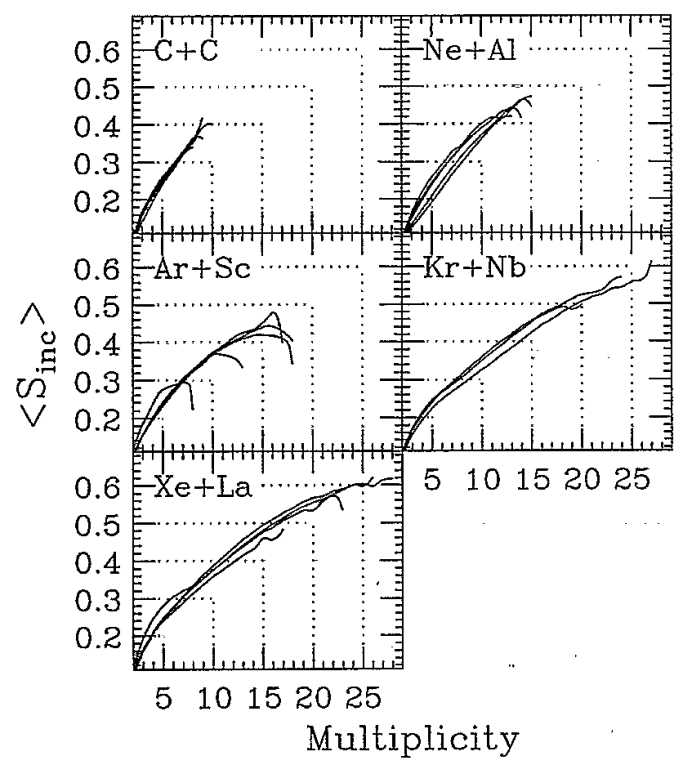

FIG. 3. The experimental semi-inclusive average sphericity as a function of the charged particle multiplicity for the reactions ${ }^{12} \mathrm{C}+{ }^{12} \mathrm{C}$ at $55,75,115$, and $155 \mathrm{MeV} /$ nucleon, ${ }^{20} \mathrm{Ne}+{ }^{27} \mathrm{Al}$ at 55 , $75,95,115$, and $135 \mathrm{MeV} /$ nucleon, ${ }^{40} \mathrm{Ar}+{ }^{45} \mathrm{Sc}$ at $15,35,75,95$, and $115 \mathrm{MeV} /$ nucleon, ${ }^{84} \mathrm{Kr}+{ }^{93} \mathrm{Nb}$ at 35,55 , and $75 \mathrm{MeV} /$ nucleon, and ${ }^{129} \mathrm{Xe}+{ }^{139} \mathrm{La}$ at $25,35,45$, and $55 \mathrm{MeV} /$ nucleon.

$b_{\max }$ is the largest impact parameter satisfying, on average, the trigger condition that was used during the data collection, which was two charged particle hits for all of the present data. According to Ref. [7], the quantity $b_{\max }$ is $\sim(0.65 \pm 0.10)\left[R_{P}+R_{T}\right]$ for the ${ }^{20} \mathrm{Ne}+{ }^{27} \mathrm{Al}$ reactions, and $\sim(0.91 \pm 0.06)\left[R_{P}+R_{T}\right]$ for the ${ }^{129} \mathrm{Xe}+{ }^{139} \mathrm{La}$ reactions.

The average sphericities of the $\sim 10 \%$ most central events selected by cuts on each of the different centrality variables are indicative of the relative strengths of the correlation between each centrality variable and the impact parameter. Two methods allowing the removal of the finite multiplicity distortions to these average sphericities will now be described. These two methods each allow the ranking of the different centrality variables in terms of the relative ability by which each actually minimizes the impact parameter in the different samples of events.

The experimental semi-inclusive average sphericity $\left\langle S_{\text {inc }}\right\rangle$ as a function of the total charged particle multiplicity is shown in Fig. 3 for the reactions ${ }^{12} \mathrm{C}+{ }^{12} \mathrm{C},{ }^{20} \mathrm{Ne}+{ }^{27} \mathrm{Al}$, ${ }^{40} \mathrm{Ar}+{ }^{45} \mathrm{Sc},{ }^{84} \mathrm{Kr}+{ }^{93} \mathrm{Nb}$, and ${ }^{129} \mathrm{Xe}+{ }^{139} \mathrm{La}$. Each frame in this figure contains the experimental dependence of the average semi-inclusive sphericity for many representative beam energies, including two extreme values, that were collected for each entrance channel. All of these curves exhibit sharp increases of the average sphericity for increasing multiplicities that result primarily from the finite multiplicity distortions.

A first method for investigating the relative efficiencies of the different centrality cuts starts with the measurement of these semi-inclusive average sphericities versus the charged particle multiplicity. $\left\langle S_{\text {inc }}\right\rangle(M)$ in a first pass through the data. Then, in a second pass, the charged particle multiplicity in each event, $M$, is used to locate the appropriate average semi-inclusive sphericity. A "reduced" sphericity is then de-

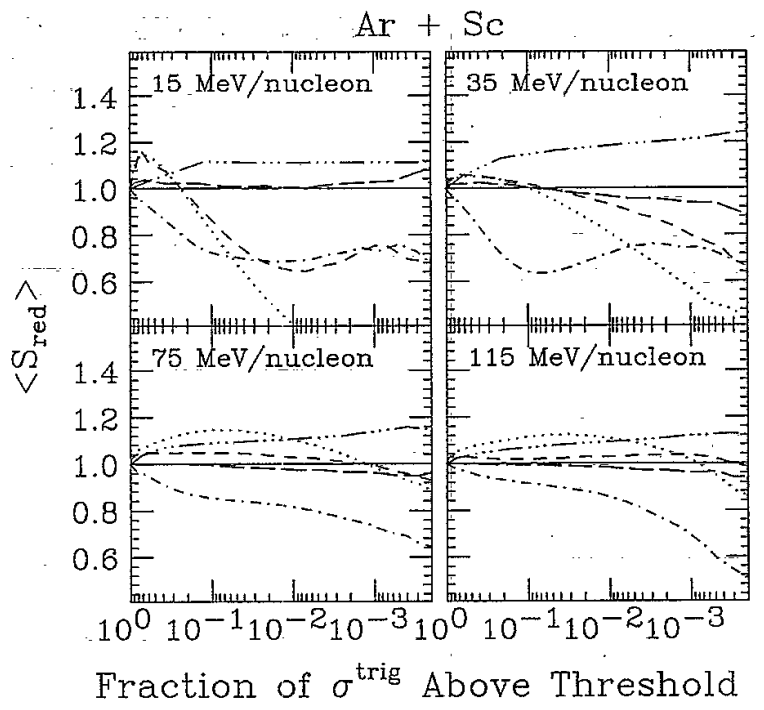

FIG. 4. The average reduced sphericity as a function of the "severity" (see text) of centrality cuts placed on $N_{\text {chgd }}$ (solid), $Z_{\mathrm{LCP}}$ (long dashed), $Z_{\mathrm{MR}}$ (short dashed), $Z_{\text {det }}$ (dot dashed), $N_{p}$ (dots dashed), and $E_{T}$ (dotted), for the ${ }^{40} \mathrm{Ar}+{ }^{45} \mathrm{Sc}$ reactions at $15,35,75$, and $115 \mathrm{MeV} /$ nucleon.

fined for each event using $S_{\text {red }}(M)=S(M) /\left\langle S_{\text {inc }}\right\rangle(M)$, where $S$ is the measured sphericity of the event. The values of these reduced sphericities averaged over the multiplicity $M$ are, on the average, free from the finite multiplicity distortions by definition. Thus the average reduced sphericities obtained by cuts on the different centrality variables imply the relative efficiencies by which these cuts minimize the impact parameters of the selected events.

The average reduced sphericity $\left\langle S_{\text {red }}\right\rangle$ as a function of the "severity" of cuts on the different centrality variables for four representative beam energies in the ${ }^{40} \mathrm{Ar}+{ }^{45} \mathrm{Sc}$ reactions is depicted in Fig. 4. The horizontal axis is defined as the probability that an event is selected versus a lower limit cut on each centrality variable separately, which corresponds to the fraction of the total trigger cross section accepted by the cut. In each frame, the average sphericities at the far left thus correspond to the impact parameter inclusive values, and proceeding horizontally to the right implies increasingly larger thresholds, i.e., increasingly strict cuts on the largest values of each centrality variable. The centrality cuts which are compared are those placed on the variables $N_{\text {chgd }}$ (solid lines), $Z_{\mathrm{MR}}$ (short dashed), $Z_{\text {det }}$ (dot dashed), $N_{p}$ (dots dashed), and $E_{T}$ (dotted). We furthermore define as a centrality variable the total charge in hydrogen and helium fragments, $\mathcal{Z}_{\mathrm{LCP}}$ (long dashed). Cuts placed on the centrality variables $N_{\text {chgd }}$ (solid lines) result in average reduced sphericities equaling unity by definition.

For the 15 and $35 \mathrm{MeV} /$ nucleon beam energies shown in Fig. 4, the largest reduced sphericities are obtained for cuts in the centrality variables $N_{p}$ and $Z_{\mathrm{LCP}}$. Relative to cuts on the other centrality variables, cuts on these variables lead to the most spherical events. At larger beam energies in this entrance channel, cuts on the total transverse kinetic energy $E_{T}$ lead to the largest sphericities.

Cuts on the variable $E_{T}$ which are stricter than $\sim 10 \%$ generally result in significantly lower average reduced sphe- 


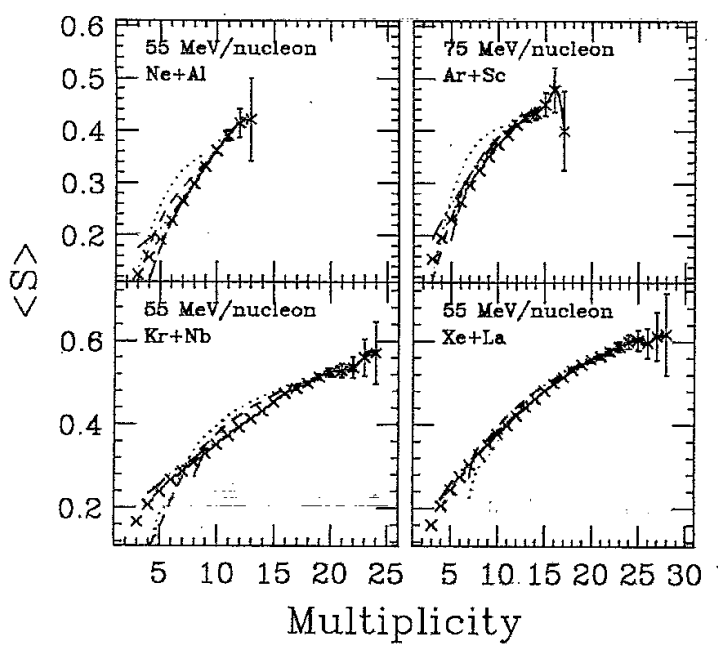

FIG. 5. The semi-inclusive average sphericities versus the multiplicity (crossed points), and the average sphericities for those events selected by one-dimensional cuts on each of the various centrality variables which accept $10 \%$ of the events (lines). The line styles are as defined above.

ricities than those when more moderate, i.e., $\sim 10 \%$, cuts are used. This behavior is independent of the entrance channel mass and the beam energy, and is attributed to a significant autocorrelation [7] between the transverse kinetic energy $E_{T}$ and the sphericity $S$, which becomes important when events with extreme values of $E_{T}$ are selected via very strict cuts. Of all of the centrality variables studied here, strict $E_{T}$ cuts have the most direct effect on the flow tensor, and hence the sphericities that are obtained, as this tensor is calculated using the Cartesian components of the particle kinetic energies.

A significant autocorrelation [7] between a given centrality variable and the sphericity would also be indicated by a relative suppression of the widths of the sphericities, $\Delta S=\sqrt{\left[\left\langle S^{2}\right\rangle-\langle S\rangle^{2}\right]}$, at specific multiplicities. In the present data, these widths depend strongly on the multiplicity (see Fig. 1), but are independent of the centrality variable upon which the cut is made for each entrance channel and beam energy when $\sim 10 \%$ cuts [19] are used. Autocorrelations [7] between $\langle S\rangle$ and the various centrality variables are thus negligible for the present (small) impact parameter cuts. Stricter cuts $(\sim 1 \%)$ were seen to lead to larger differences between the widths of the sphericities of the events accepted by cuts on each of the different centrality variables (especially $E_{T}$ ), and hence significant autocorrelations between the average sphericities and the various centrality variables. Such autocorrelations significantly suppress the average sphericities in the selected events.

An alternative method for extracting the relative efficiencies of the various centrality variables for the selection of central events is now described. The average sphericities of the events passing the one-dimensional $\sim 10 \%$ cuts [19] described above are depicted in Fig. 5 versus the charged particle multiplicity. The different line styles correspond to cuts on each of the different centrality variables that were shown in Fig. 4, while the semi-inclusive average sphericities, i.e., from Fig. 3, are shown as the crossed points. Four representative entrance channels and beam energies are shown:

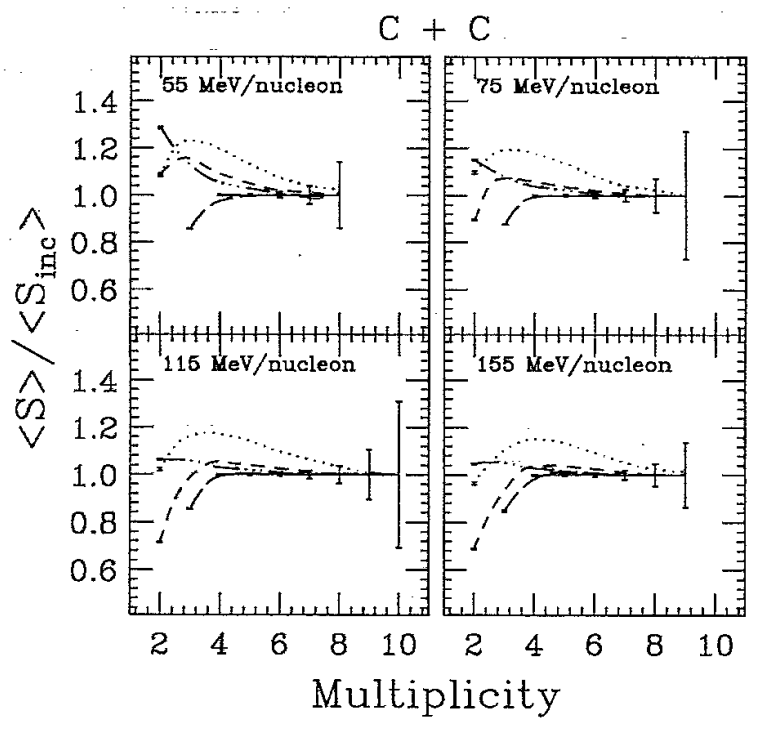

FIG. 6. The ratios of the average sphericities selected by $\sim 10 \%$ cuts on each of the various centrality variables to the semiinclusive average sphericities as a function of the multiplicity for the ${ }^{12} \mathrm{C}+{ }^{12} \mathrm{C}$ reactions at beam energies of $55,75,115$, and 155 $\mathrm{MeV} /$ nucleon.

${ }^{20} \mathrm{Ne}+{ }^{27} \mathrm{Al}$ at $55 \mathrm{MeV} /$ nucleon, ${ }^{40} \mathrm{Ar}+{ }^{45} \mathrm{Sc}$ at $75 \mathrm{MeV} /$ nucleon, ${ }^{84} \mathrm{Kr}+{ }^{93} \mathrm{Nb}$ at $55 \mathrm{MeV} /$ nucleon, and ${ }^{129} \mathrm{Xe}+{ }^{139} \mathrm{La}$ at $55 \mathrm{MeV} /$ nucleon. Significant differences between the average sphericities at specific multiplicities obtained from cuts on the different centrality variables are apparent.

To clarify these differences by removing the large variation resulting primarily from the finite multiplicity distortions, the ratios of the average sphericities in each sample of selected events (lines) and the semi-inclusive average sphericities (crossed points) are formed at each experimental multiplicity. Figures 6 through 10 depict these ratios for four representative beam energies each in the ${ }^{12} \mathrm{C}+{ }^{12} \mathrm{C}$,

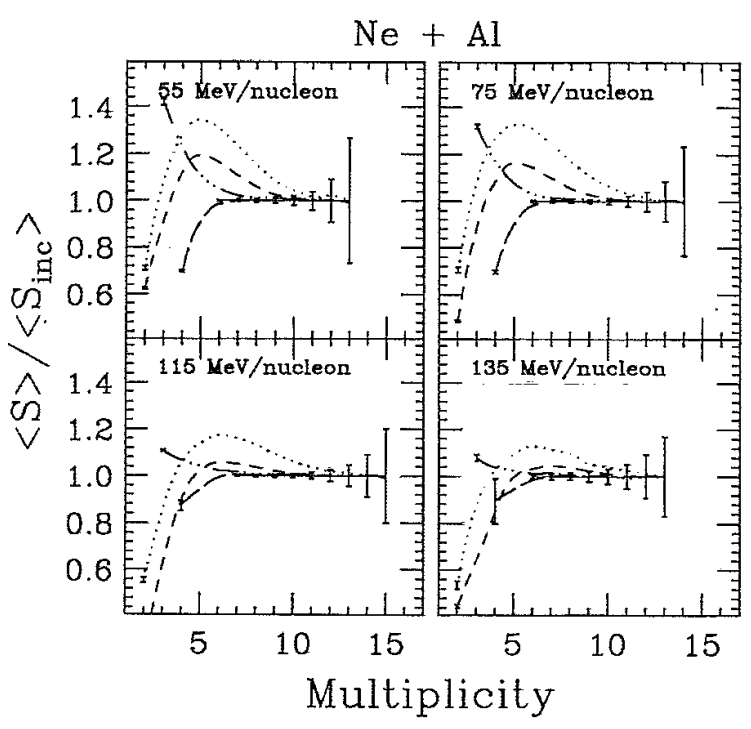

FIG. 7. The same as Fig. 6 , but for the ${ }^{20} \mathrm{Ne}+{ }^{27} \mathrm{Al}$ reactions at $55,75,95$, and $115 \mathrm{MeV} /$ nucleon. 


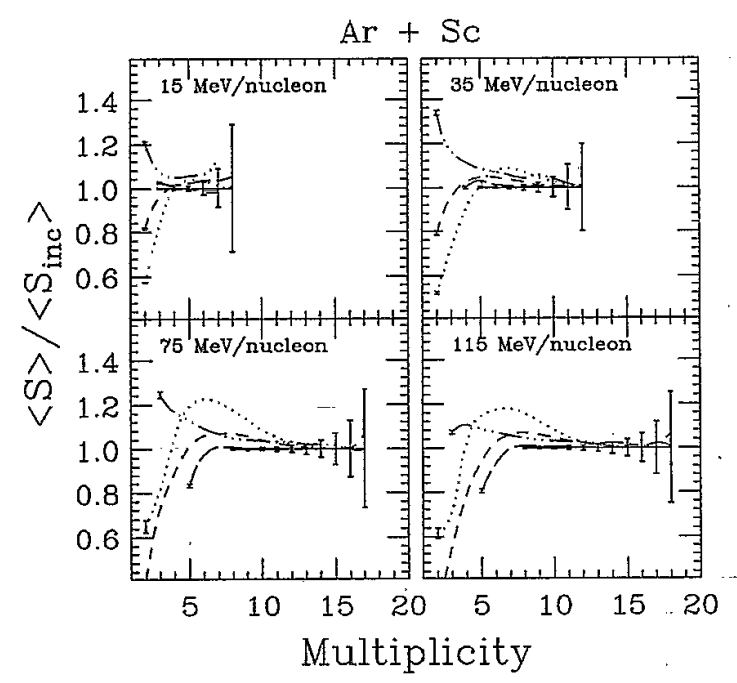

FIG. 8. The same as Fig. 6, but for the ${ }^{40} \mathrm{Ar}+{ }^{45} \mathrm{Sc}$ reactions at $15,35,75$, and $115 \mathrm{MeV} /$ nucleon.

${ }^{20} \mathrm{Ne}+{ }^{27} \mathrm{Al},{ }^{40} \mathrm{Ar}+{ }^{45} \mathrm{Sc},{ }^{84} \mathrm{Kr}+{ }^{93} \mathrm{Nb}$, and ${ }^{129} \mathrm{Xe}+{ }^{139} \mathrm{La}$ entrance channels, respectively. By definition, the ratios of the average sphericities from an $N_{\text {chgd }}$ cut and the semi-inclusive average sphericities is unity. The cuts on the other centrality variables generally allow events extending to lower multiplicities than those selected by the cuts on $N_{\text {chyd }}$. The statistical error bars in these ratios are not plotted for every curve and each multiplicity for clarity. Only the errors for the $N_{\text {chgd }}$ cuts (solid) and the errors for the lowest multiplicity accepted by each of the other centrality cuts are drawn.

In the ${ }^{12} \mathrm{C}+{ }^{12} \mathrm{C}$ reactions at $55,75,115$, and $155 \mathrm{MeV} /$ nucleon (Fig. 6), significantly different average reduced sphericities are obtained from small impact parameter cuts on the different centrality variables. The average reduced sphericities from the $E_{T}$ cuts are generally the largest for all of the beam energies shown. Similar trends are visible in the ${ }^{20} \mathrm{Ne}+{ }^{27} \mathrm{Al}$ (Fig. 7), and the ${ }^{40} \mathrm{Ar}+{ }^{45} \mathrm{Sc}$ (Fig. 8) reactions for the same range of beam energies. However, at the lower

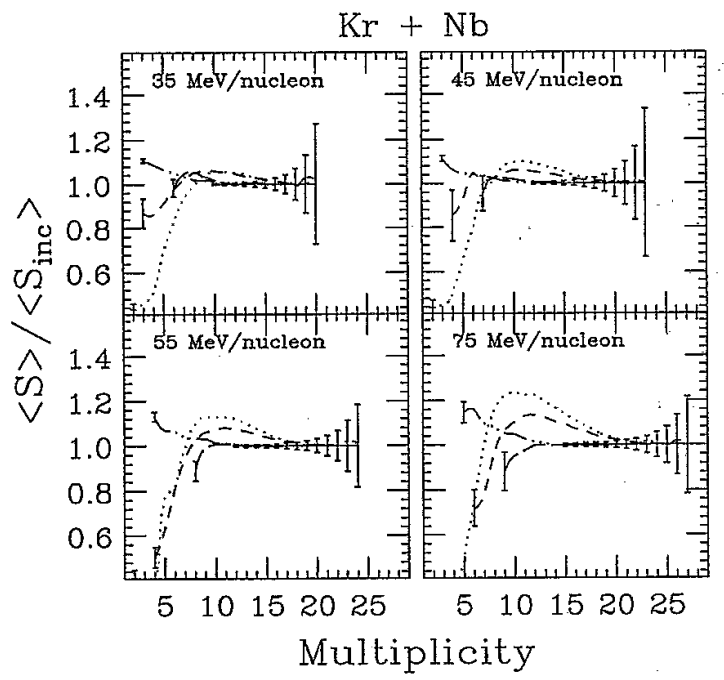

FIG. 9. The same as Fig. 6, but for the ${ }^{84} \mathrm{Kr}+{ }^{93} \mathrm{Nb}$ reactions at $35,45,55$, and $75 \mathrm{MeV} /$ nucleon.

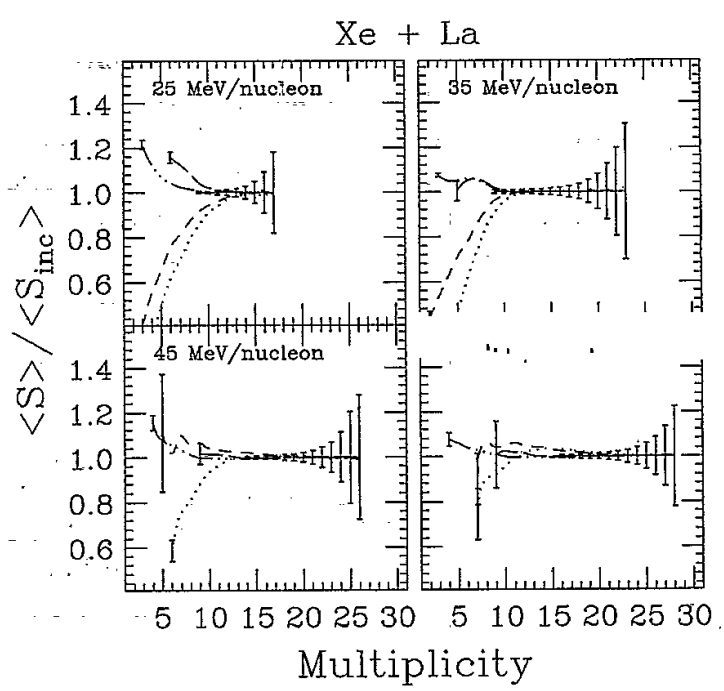

FIG. 10. The same as Fig. 6, but for the ${ }^{129} \mathrm{Xe}+{ }^{139} \mathrm{La}$ reactions at $25,35,45$, and $55 \mathrm{MeV} /$ nucleon.

beam energies in the ${ }^{40} \mathrm{~A} . \mathrm{r}+{ }^{45} \mathrm{Sc}$ entrance channel, the largest reduced sphericities are obtained from the cuts on the proton multiplicity $N_{p}$. As the entrance channel mass increases, i.e., for the ${ }^{84} \mathrm{Kr}+{ }^{93} \mathrm{Nb}$ and ${ }^{129} \mathrm{Xe}+{ }^{139} \mathrm{La}$ reactions (Figs. 9 and 10), the differences between the average reduced sphericities obtained from the different centrality cuts decreases, as compared to the lighter entrance channels at the same beam energies. The relative efficiencies of the different small impact parameter cuts therefore become similar as the entrance channel mass is increased.

To summarize the results of Figs. 6 through 10 in a way that accounts for the different weighting of each of these curves by the different spectra of total charged particle multiplicities selected using each cut, the averages over each of

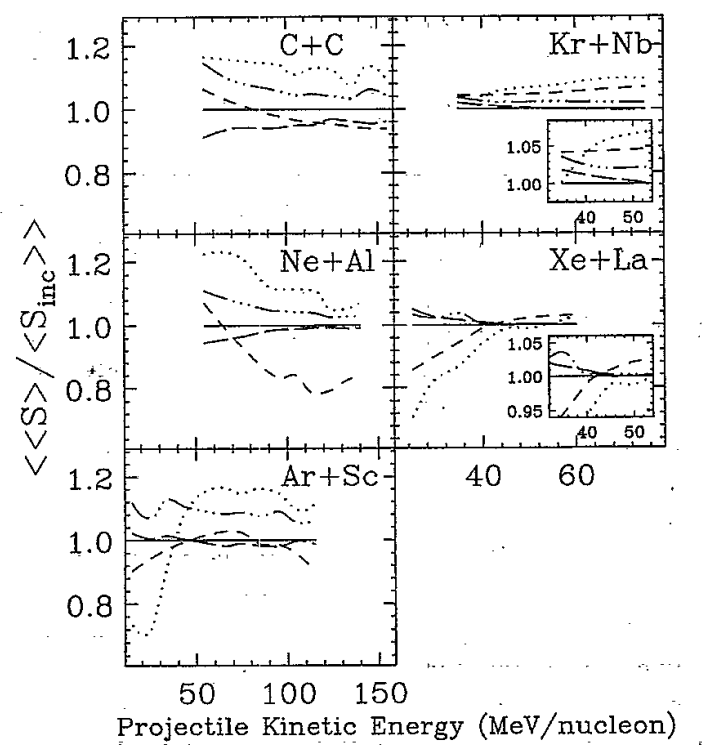

FIG. 11. The weighted averages over the multiplicity of the curves shown in Figs. 6 to 10 versus the projectile kinetic energy. Expanded views of the ${ }^{84} \mathrm{Kr}+{ }^{93} \mathrm{Nb}$ and ${ }^{129} \mathrm{Xe}+{ }^{139} \mathrm{La}$ results are inset for clarity. 
these curves are formed using weights given by the number of events in each bin of this selected multiplicity. As the finite multiplicity distortions have already been removed on the average from Figs. 6 to 10, this weighted averaging over these curves is not affected by these distortions. The relative ordering of the weighted averages of the curves shown in Figs. 6 to 10 is therefore indicative of the relative efficiencies of the various centrality variables for the selection of small impact parameter events.

These weighted averages are shown in Fig. 11 as a function of the beam energy for all five entrance channels. All of the available beam energies were included in this figure. The subtle variations along some of these curves in this figure are an artifact of that noted in Ref. [19]; the statistical uncertainty in this figure is negligible. In the two lightest entrance channels, ${ }^{12} \mathrm{C}+{ }^{12} \mathrm{C}$ and ${ }^{20} \mathrm{Ne}+{ }^{27} \mathrm{Al}$, the largest weighted averages are obtained from cuts on $E_{T}$ and $N_{p}$ for all of the available beam energies ( $E_{\text {proj }} \geqslant 55 \mathrm{MeV} /$ nucleon). Transitions in the ordering of the relative efficiencies of the various centrality cuts are observed as a function of the beam energy in the ${ }^{40} \mathrm{Ar}+{ }^{45} \mathrm{Sc},{ }^{84} \mathrm{Kr}+{ }^{93} \mathrm{Nb}$, and ${ }^{129} \mathrm{Xe}+{ }^{139} \mathrm{La}$ reactions. In each of these entrance channels, the smallest impact parameter events for beam energies below about $45 \mathrm{MeV} /$ nucleon are most efficiently selected using cuts on the variables $N_{p}$ and $Z_{\text {LCP. }}$. Above this beam energy, the most efficient pairs of centrality variables are $\left(E_{T}, N_{p}\right)$, $\left(E_{T}, Z_{\mathrm{MR}}\right)$, and $\left(Z_{\mathrm{MR}}, E_{T}\right)$, for the ${ }^{40} \mathrm{Ar}+{ }^{45} \mathrm{Sc},{ }^{84} \mathrm{Kr}+{ }^{93} \mathrm{Nb}$, and ${ }^{129} \mathrm{Xe}+{ }^{139} \mathrm{La}$ reactions, respectively.

The beam-energy-dependent transitions in the most efficient means of selecting the central events seen in Fig. 11 are assumed to be related to similarly beam-energy-dependent transitions in the predominant reaction mechanisms at small impact parameters. Copious light particle emission is expected from excited systems decaying by a sequential binary mechanism, due to the importance of the Coulomb and angular momentum barriers during such decays. Thus one would expect that the most central collisions for an entrance channel and beam energy for which SB disassembly dominates would be those for which the largest light particle multiplicities were observed. A generally different reaction mechanism, i.e., one exhibiting distinct topological differences relative to $\mathrm{SB}$ events, might best be classified using centrality variables differing from those that best classify reactions leading to SB disassembly. The extent to which the transitions apparent in Fig. 9 are indeed an artifact of transitions from SB to MF disassembly in the small impact parameter collisions is further investigated in the following sections. Events were generated using a number of SB and $\mathrm{MF}$ model codes and filtered by the software reproduction of the apparatus, allowing direct comparisons to the experimental central events.

It is noted that topological fluctuations in the events result in the allowance of some range of impact parameters for a specific value of an experimentally measured centrality variable. Events in which such fluctuations result in an underestimation of the impact parameter are a clear source of bias for one-dimensional centrality cuts. The use of twodimensional centrality cuts would limit the contributions of such events [17]. Such a cut will henceforth be applied. These cuts select events in which the two most efficient centrality variables (from Fig. 11) exceed the $\sim 10 \%$ thresholds
[19] used above. Generally, $\sim 4-8 \%$ of the minimum bias events $\left(\langle b\rangle / b_{\max } \sim 0.2-0.28\right.$ geometrically) are accepted.

\section{COMPARISONS TO MODEL EVENTS}

In this section, events generated by a number of different model codes which describe either a SB or MF disassembly mechanism are filtered through the detailed software replica of the MSU 4 $\pi$ Array and compared directly to the data. The event generation was performed in both dynamic (FREESCO [9]) and hybrid approaches [7], for which the BoltzmannUehling-Uehlenbeck (BUU) [20] and QMD [21] models were used to describe the initial stages of the reactions. These codes were run with the default parameters with the exception of the charge, mass, and excitation energy in the composite system. This information was extracted from the BUU calculations in the same manner as described in Refs. [7] and [22]. A soft equation of state was assumed, and the calculations were terminated when the radial density profile of the composite system most closely resembled that of a ground state nucleus [20]. These calculations were performed at an impact parameter of $b=0.2\left[R_{P}+R_{T}\right]$.

The "after-burners" used in the hybrid event generation were the BERLIN [23] and COPENHAGEN $[2,24]$ MF codes, as well as the SB codes GEMINI [25] and SEQUENTIAL [26]. The BERLIN and GEMINI codes require the input of a cutoff angular momentum, which was taken as the maximum angular momentum that can be supported by the composite system predicted by the BUU calculations for each entrance channel and beam energy. These are in the range from $\sim 36 \hbar$ to $\sim 80 \hbar$, depending on the reaction.

A more detailed description of the models and the present event generation can be found in Ref. [7]. The MF models, BERLIN and COPENHAGEN, assume an initially expanded and spherical freeze-out volume. The particle emission probabilities and patterns depend on the available phase space and the strong interparticle Coulomb repulsions. Secondary decays of prefragments are included in the calculation. The SB models, GEMINI and SEQUENTIAL, describe emission patterns that are relatively more elongated $[9,13]$, due to the assumed series of momentum conserving two-body decay steps following an initial (a)symmetric fission.

The excitation energies expected for the present central reactions on the basis of the BUU calculations are in the range from $\sim 3$ to $\sim 30 \mathrm{MeV} /$ nucleon [7]. The SB models are thus in a regime where the disassembly involves some number of fissionlike mass splits and particle evaporation. The number of these fissionlike steps increases monotonically with the beam energy in the SB picture (Casini et al. [27]). The average sphericities of such compound binary events are still less than those for MF events of the same multiplicity, as the fragments resulting from the fission of a fission fragment conserve the momentum of the decaying fragment. However, as the number of fission steps, i.e., the excitation energy, is increased, the initial elongation of the $\mathrm{SB}$ events becomes more and more obscured. The average sphericities of SB events should therefore approach those for $\mathrm{MF}$ events as the beam energy is increased.

While the initial freeze-out volume in the MF models is spherical by definition, the average sphericities of the events generated by these models need not be consistent with the 
maximum sphericity possible for each multiplicity. Coulomb repulsions strongly affect the trajectories of the primordial particles, and secondary emission occurs in flight.

With increasing excitation energies, the MF codes predict an evolution in the mass distributions that is similar to that obtained from the SB models. One might therefore expect that the MF models should provide an accurate description of the event shapes for all excitation energies. The experimental results presented below will answer this question. We note, however, that the fragment momenta in the two classes of models are the result of two very different theoretical pictures for the disassembly. While the MF and SB models may result in similar mass distributions for some system mass and excitation energy, one should not expect that the average sphericities from the two classes of models are also similar (at the same multiplicity).

For a given excited nucleus and at a specific final state multiplicity, the values of $\langle S\rangle$ for the events generated using the SEQUENTIAL ( BERLN) code should agree with those from the GEMINI (COPENHAGEN) codes, and this was found to be true to about the $\sim 15 \%$ level. This should be taken as an estimate of the systematic uncertainty of the present model calculations. For clarity in the figures below, only the results using the MF code BERLIN [23] and SB code SEQUENTIAL [26] will be plotted. The average sphericities from the dynamic MF model FREESCO and the hybrid MF models QMD + COPENHAGEN and BUU + COPENHAGEN also agree to within $\sim 15 \%$ at specific multiplicities, implying a relative insensitivity of the present shape comparisons to the model chosen for the description of the initial stages of the reactions.

As opposed to the analyses in the previous section, the finite multiplicity distortions to the average sphericities are removed from the results in this and the following section graphically. The sphericities from the data and the model calculations will be plotted as a function of the number of particles included in the calculation of the flow tensor. Comparisons between the experimental and model results are thus performed at specific values of this multiplicity, and are hence free from the finite multiplicity distortions.

By assumption, the average sphericities of the selected experimental events cannot be below the predictions of the SB model calculations, or above the predictions of the MF models, within the $\sim 15 \%$ systematic uncertainties. An increase in the average sphericity, relative to the predictions of the SB models, towards the predictions of the MF models with increases in the beam energy is indicative of an evolution of the reaction mechanism from SB disassembly to MF.

The average sphericities of the central experimental(generated) events are plotted versus the measured(filtered) total charged particle multiplicity in Figs. 12, 13, and 14 for the ${ }^{40} \mathrm{Ar}+{ }^{45} \mathrm{Sc},{ }^{84} \mathrm{Kr}+{ }^{93} \mathrm{Nb}$, and ${ }^{129} \mathrm{Xe}+{ }^{139} \mathrm{La}$ reactions respectively. The crossed points depict the results for the central experimental events, while the solid(dashed) lines are the results from the filtered $\mathrm{MF}(\mathrm{SB})$ model events. The various lines depicting the theoretical predictions are drawn only over a region for which the statistical uncertainty in the simulations is less than $20 \%$. For each reaction, the number of events generated using each model is the same as the number of experimental central events.

The average sphericities of the central ${ }^{40} \mathrm{Ar}+{ }^{45} \mathrm{Sc}$ reac-

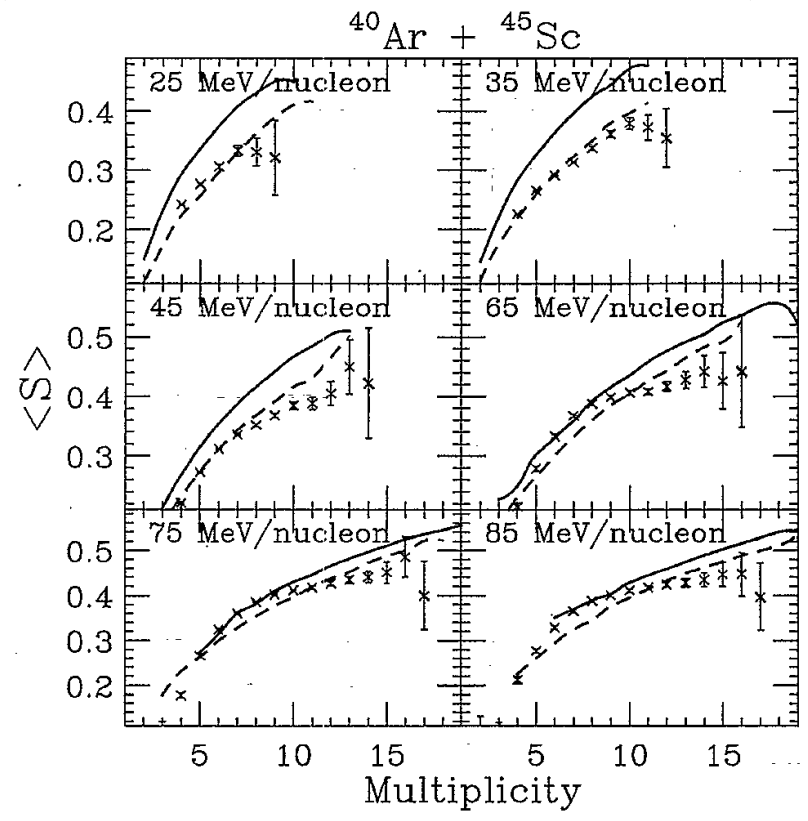

FIG. 12. The average sphericity versus the total charged particle multiplicity for central ${ }^{40} \mathrm{Ar}+{ }^{45} \mathrm{Sc}$ reactions at beam energies of 25 , $35,45,65,75$, and $85 \mathrm{MeV} /$ nucleon. The experimental values are shown as the crossed points, while the solid (dashed) lines depict the predictions of the filtered MF (SB) models.

tions at beam energies near and below $45 \mathrm{MeV} /$ nucleon are in agreement with those predicted by the SB codes. The data approach the MF predictions for multiplicities on the order of 8 between the beam energies of 45 and $65 \mathrm{MeV} /$ nucleon. At larger multiplicities in this entrance channel, a significant suppression to below the SB model predictions is visible. This implies a failure in one or more of the assumptions used in the present event generation for these high multiplicity

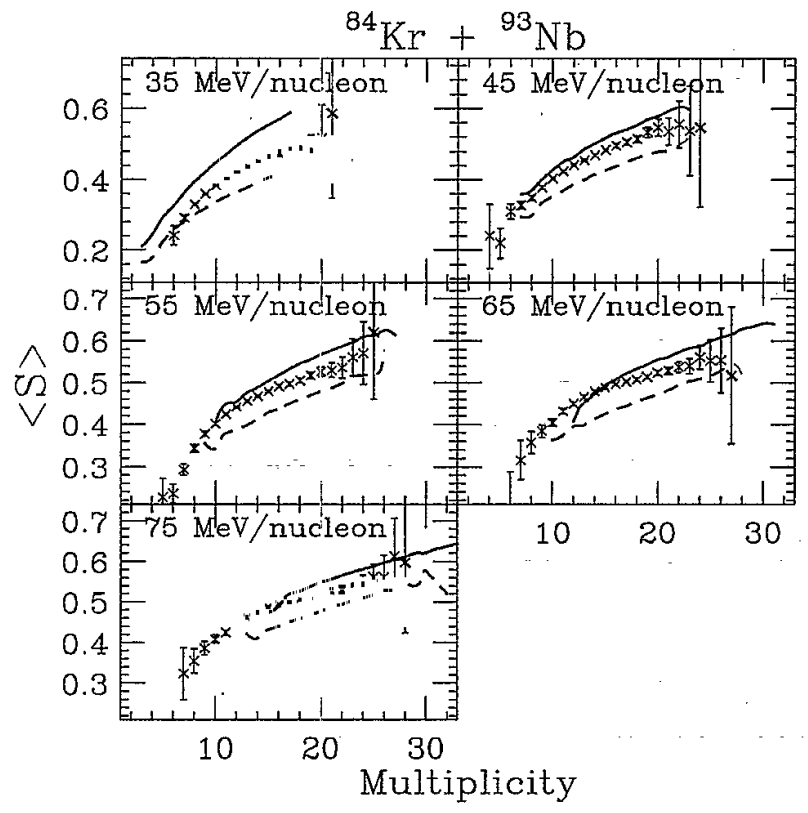

FIG. 13. The same as Fig. 12, but for the ${ }^{84} \mathrm{Kr}+{ }^{93} \mathrm{Nb}$ reactions at $35,45,55,65$, and $75 \mathrm{MeV} /$ nucleon. 


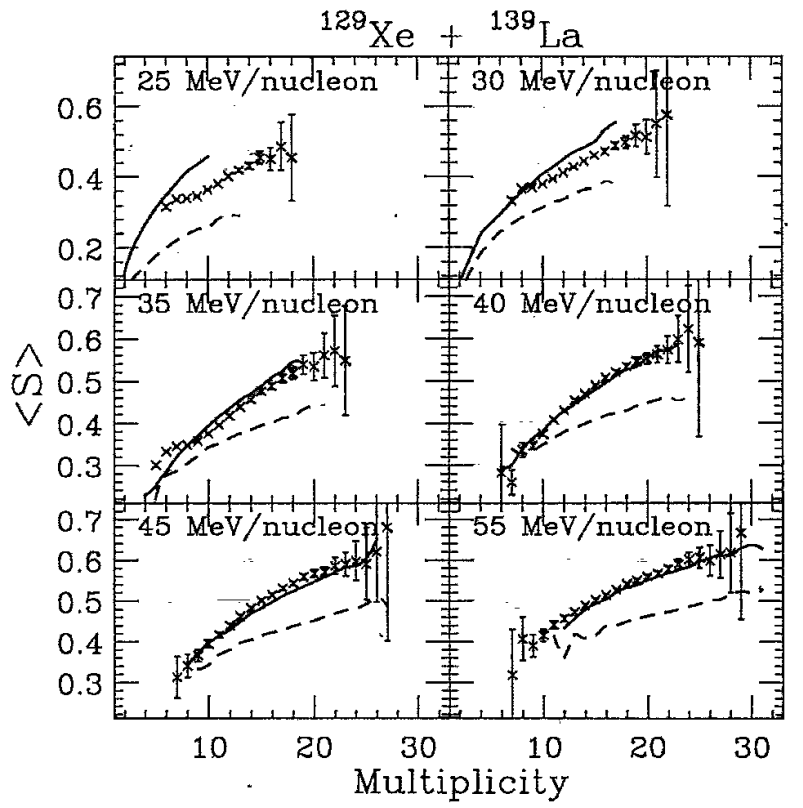

FIG. 14. The same as Fig. 12, but for the ${ }^{129} \mathrm{Xe}+{ }^{139} \mathrm{La}$ reactions at $25,30,35,40,45$, and $55 \mathrm{MeV} /$ nucleon.

events in this entrance channel. This suppression is further investigated in the next section.

In the central ${ }^{84} \mathrm{Kr}+{ }^{93} \mathrm{Nb}$ and ${ }^{129} \mathrm{Xe}+{ }^{139} \mathrm{La}$ reactions, the average sphericities from the data are always between the predictions of the filtered SB and MF models for all available beam energies. For the central ${ }^{129} \mathrm{Xe}+{ }^{139} \mathrm{La}$ reactions (Fig. 14), the average sphericities increase, relative to the SB predictions, with the increases in the beam energy. For beam energies near and above $\sim 40 \mathrm{MeV} /$ nucleon, the experimental average sphericities are in rather remarkable agreement with the MF model predictions over the entire range of multiplicities. Similar increases in the average sphericities of the central ${ }^{84} \mathrm{Kr}+{ }^{93} \mathrm{Nb}$ events (Fig. 13) are also observed, although the model calculations tend to overpredict the total charged particle multiplicities for these reactions.

At a specific multiplicity, the predictions of the SB and MF models converge as the beam energy is increased. For example, in the central ${ }^{40} \mathrm{Ar}+{ }^{45} \mathrm{Sc}$ reactions at a multiplicity of 10 , the predicted difference in average sphericity between the SB and MF models decrease from $\sim 0.07$ to $\sim 0.03$ as the beam energy is increased from 25 to $85 \mathrm{MeV} /$ nucleon. The sensitivity by which one can distinguish between the SB and MF scenarios using the average sphericities thus decreases monotonically as the beam energy is increased.

The results shown in Figs. 13 and 14 for the central ${ }^{84} \mathrm{Kr}+{ }^{93} \mathrm{Nb}$ and ${ }^{129} \mathrm{Xe}+{ }^{139} \mathrm{La}$ reactions are shown versus the beam energy for specific multiplicities in Figs. 15 and 16, respectively. The error bars depict the statistical uncertainties only. The MF(SB) model predictions are shifted by +1 $(-1) \mathrm{MeV} /$ nucleon along the abscissa for clarity, and shown as the solid(dashed) lines as in the previous figures.

The MF model predictions for the central ${ }^{84} \mathrm{Kr}+{ }^{93} \mathrm{Nb}$ $\left({ }^{129} \mathrm{Xe}+{ }^{139} \mathrm{La}\right)$ reactions decrease with increases in the beam energy up to $\sim 50(40) \mathrm{MeV} / n u c l e o n$, and are consistent with a constant value for larger beam energies. The SB model predictions rise, and then become constant, for increasing

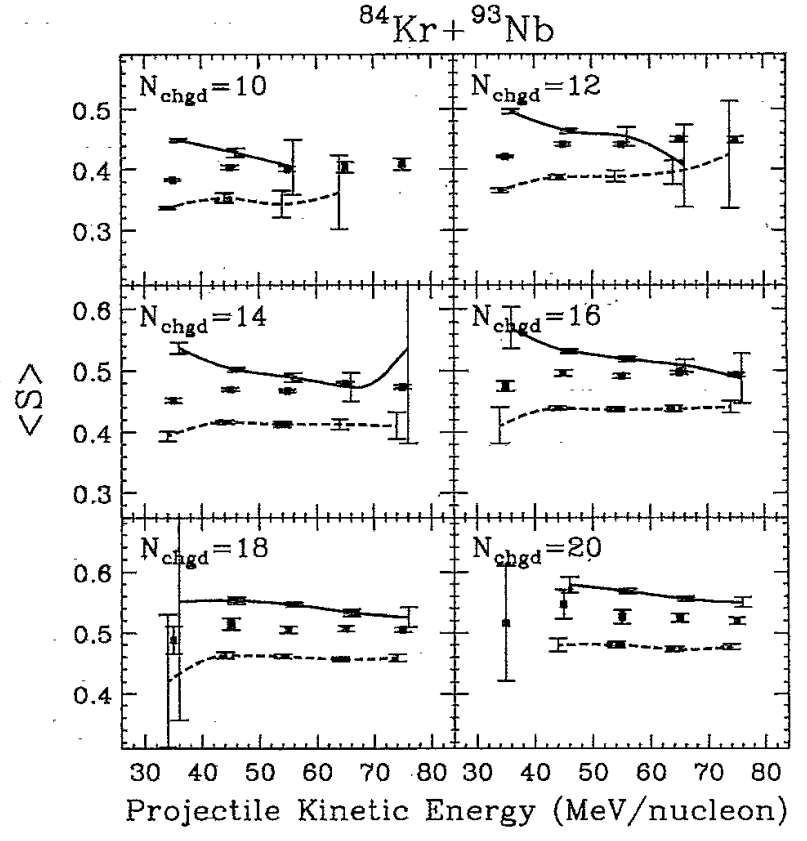

FIG. 15. The average sphericity versus the beam energy for the central ${ }^{84} \mathrm{Kr}+{ }^{93} \mathrm{Nb}$ reactions at specific values of the total charged particle multiplicity as labeled in each frame. The SB(MF) model results are shown as the dashed(solid) lines. The error bars are statistical, and the abscissa for the model predictions have been shifted by $\pm 1 \mathrm{MeV}$ for clarity.

energies. For the ${ }^{84} \mathrm{Kr}+{ }^{93} \mathrm{Nb}$ reactions shown in Fig. 15, the experimental results are consistent with the MF model predictions for multiplicities less than $\sim 15$ and beam energies near and above $\sim 50 \mathrm{MeV} /$ nucleon. At a specific multiplicity, the experimental sphericities decrease, i.e., proceed towards

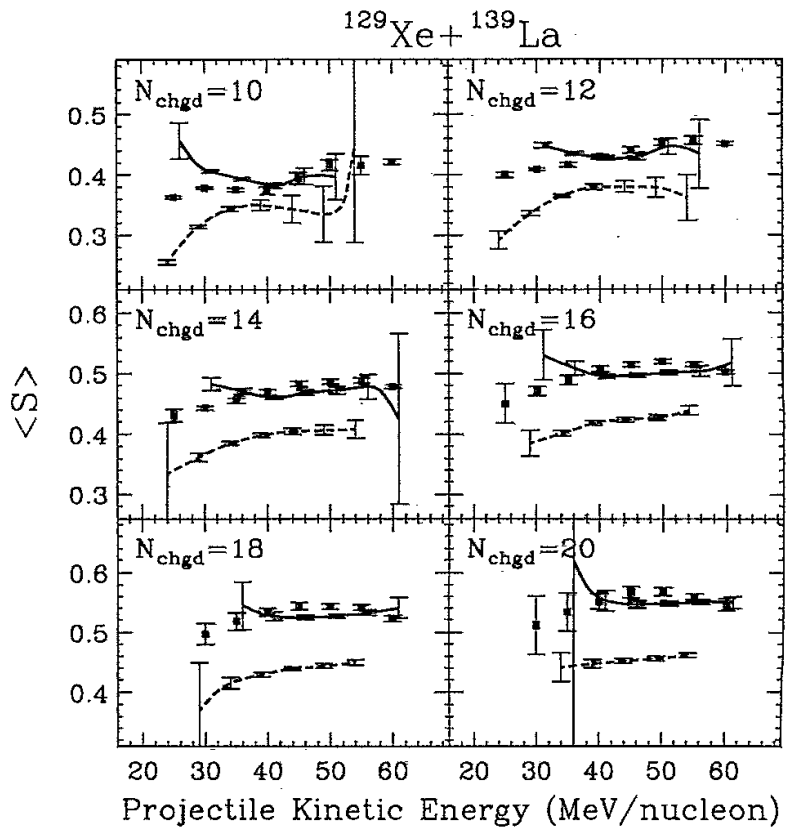

FIG. 16. The same as Fig. 15, but for the central ${ }^{129} \mathrm{Xe}+{ }^{139} \mathrm{La}$ reactions. 


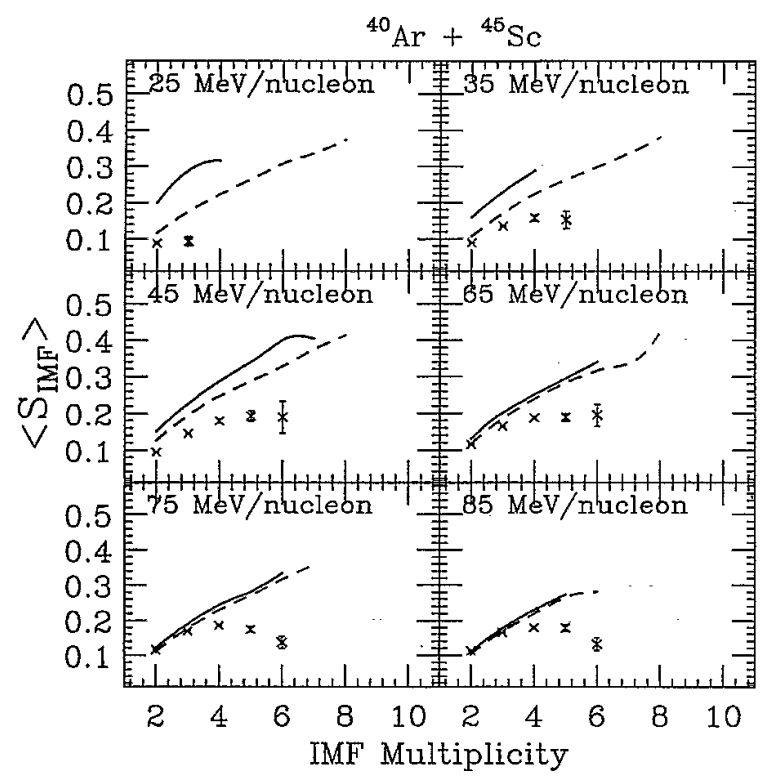

FIG. 17. The average IMF sphericity versus the total IMF multiplicity for central ${ }^{40} \mathrm{Ar}+{ }^{45} \mathrm{Sc}$ reactions at beam energies of 25,35 , $45,65,75$, and $85 \mathrm{MeV} /$ nucleon. The experimental values are shown as the crossed points, while the solid (dashed) line depict the predictions of the filtered MF (SB) models.

the SB predictions, as the beam energy is decreased. As the multiplicity is increased, the beam energy at which the data come to agreement with the MF predictions increases. At the highest multiplicities, the data are between the SB and MF predictions for all beam energies. Similar trends are observed for the central ${ }^{129} \mathrm{Xe}+{ }^{139} \mathrm{La}$ reactions. For all multiplicities, the data are between the SB and MF model predictions for beam energies less than $\sim 40 \mathrm{MeV} /$ nucleon. For higher beam energies, the experimental average sphericities are equal to, or in slight excess of, those predicted by the MF models.

\section{SUBSET SHAPES}

In this section, the sphericity of particular subsets of an event will be distinguished from the sphericity of all of the particles in the event. This will allow additional tests of the accuracy by which the present model calculations reproduce the average sphericities of the experimental events. It is important to note that the sphericities of such event subsets are affected to some degree by the fact that the total momentum of a particular subset need not be conserved. Another source of bias to the subset sphericities would clearly contribute if the subset was defined on the basis of some kinematical aspect of the event. The simulations described in the previous section indicate, however, that reasonably general definitions of subsets based on the particle charges do not suffer from significant bias from either of these effects. Examples of two such subsets are the light charged particles (LCP's) and intermediate mass fragments (IMF's). Light particle emission is probable throughout the reaction, including that from the preequilibrium stages as well as in secondary decays of excited fragments far from the collision zone and long after freeze-out. The emission of IMF's, on the other hand, is more a reflection of the excited composite system and its

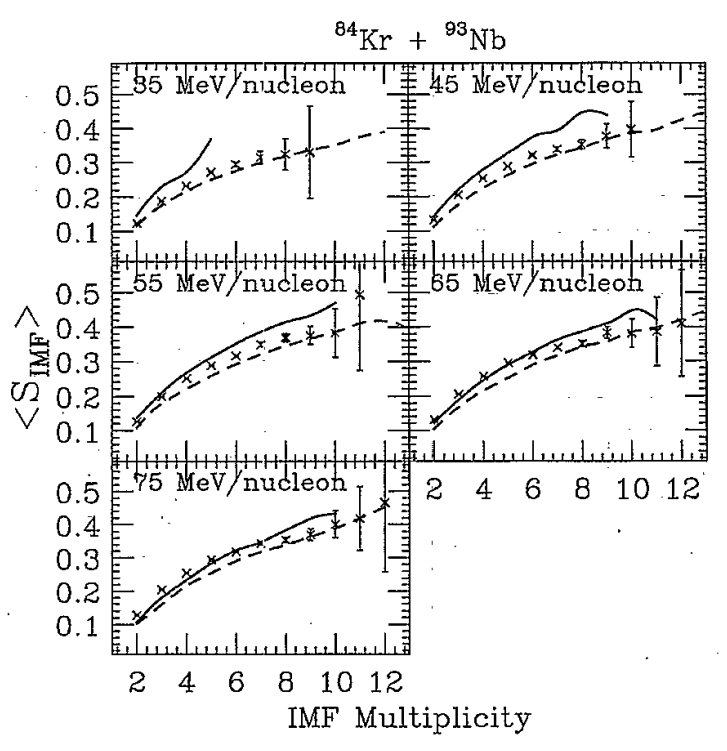

FIG. 18. The same as Fig. 17, but for the ${ }^{84} \mathrm{Kr}+{ }^{93} \mathrm{Nb}$ reactions at $35,45,55,65$, and $75 \mathrm{MeV} /$ nucleon.

expansion to low densities, so we concentrate on the average sphericities of the IMF's in this section.

The previous section noted that the average sphericities of the largest multiplicity central events in the ${ }^{40} \mathrm{Ar}+{ }^{45} \mathrm{Sc}$ (and lighter) reactions were significantly below the predictions of the present SB model calculations. For all of the available entrance channels and beam energies, the largest average multiplicities of IMF's are found in the events with the largest total charged particle multiplicities [7]. As depicted in Fig. 17, the average IMF sphericities $\left\langle S_{\mathrm{IMF}}\right\rangle$ are generally well below those predicted by the filtered SB model calculations for all IMF multiplicities for the central ${ }^{40} \mathrm{Ar}+{ }^{45} \mathrm{Sc}$ reactions. As the emission of IMF's is most important at the largest charged particle multiplicities, these suppressed IMF sphericities effect the suppression at large charged particle multiplicities seen in Fig. 12. A similar suppression of the IMF sphericities to below the SB model predictions was also observed for the central ${ }^{12} \mathrm{C}+{ }^{12} \mathrm{C}$ and ${ }^{20} \mathrm{Ne}+{ }^{27} \mathrm{Al}$ reactions. We will return to a discussion of this suppression of the IMF sphericities for these (light) entrance channels near the end of this section.

The IMF sphericities in the central ${ }^{84} \mathrm{Kr}+{ }^{93} \mathrm{Nb}$ reactions are in good agreement with the SB model predictions for the $35 \mathrm{MeV} /$ nucleon data (Fig. 18). They increase, relative to the SB model predictions, with increases in the beam energy, coming to quantitative agreement with the MF model predictions for beam energies near and above $65 \mathrm{MeV} /$ nucleon. For the central ${ }^{129} \mathrm{Xe}+{ }^{139} \mathrm{La}$ reactions (shown in Fig. 19); the average IMF sphericities are always between the SB and MF model predictions. Good agreement between the experimental IMF sphericities and the MF model predictions is observed for beam energies above $\sim 40 \mathrm{MeV} /$ nucleon.

The beam energy dependence of the average IMF sphericities is shown in Fig. 20 for specific IMF multiplicities in the central ${ }^{40} \mathrm{Ar}+{ }^{45} \mathrm{Sc},{ }^{84} \mathrm{Kr}+{ }^{93} \mathrm{Nb}$, and ${ }^{129} \mathrm{Xe}+{ }^{139} \mathrm{La}$ reactions. Some points in the upper frame of Fig. 20 have been vertically offset by the amounts shown for clarity, while the solid lines in all three frames are included only to guide the eye. The points are also offset by $\pm 1 \mathrm{MeV} /$ nucleon along 


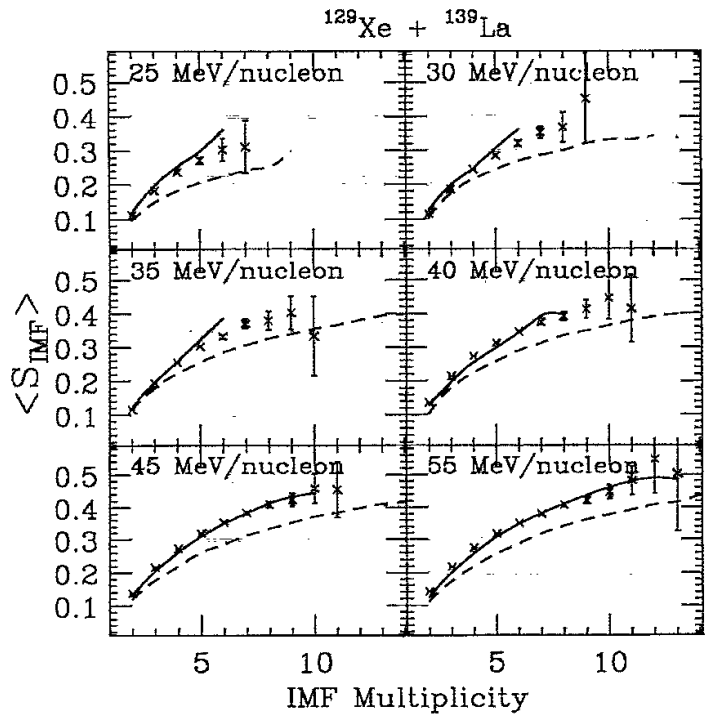

FIG. 19. The same as Fig. 17, but for the ${ }^{129} \mathrm{Xe}+{ }^{139} \mathrm{La}$ reactions at $25,30,35,40,45$, and $55 \mathrm{MeV} /$ nucleon.

the abscissa for the different values of $N_{\text {IMF }}$ so that the error bars are clearly visible. In the central ${ }^{40} \mathrm{Ar}+{ }^{45} \mathrm{Sc}$ reactions, a significant increase in the average IMF sphericities is observed at beam energies near $55 \mathrm{MeV} /$ nucleon for all IMF multiplicities. A similar increase of the IMF sphericities is also observed in the central ${ }^{129} \mathrm{Xe}+{ }^{139} \mathrm{La}$ reactions at beam energies near $40 \mathrm{MeV} /$ nucleon. In the ${ }^{84} \mathrm{Kr}+{ }^{93} \mathrm{Nb}$ entrance channel, the average IMF sphericity for each value of $N_{\text {IMF }}$ increases when going from 35 to $45 \mathrm{MeV} /$ nucleon in beam energy, and is constant for beam energies from 45 to 75 $\mathrm{MeV} /$ nucleon. Similar maxima in the average sphericities of all of the particles in the central events can be seen in Figs. 15 and 16 at beam energies similar to those leading to maximal IMF sphericities.

The beam energy dependence of the IMF sphericities in the central ${ }^{84} \mathrm{Kr}+{ }^{93} \mathrm{Nb}\left({ }^{129} \mathrm{Xe}+{ }^{139} \mathrm{La}\right.$ ) reactions is compared to the SB and MF model predictions in Fig. 21 (22). Like the predicted sphericities of entire events shown in Figs. 15 and 16 , the SB (MF) model predictions for the IMF sphericities rise (fall) with increasing beam energies up to a certain point, then remain consistent with a constant value. For all IMF multiplicities, the IMF sphericities in the ${ }^{84} \mathrm{Kr}+{ }^{93} \mathrm{Nb}$ reactions are equal to or slightly in excess of the SB (MF) model predictions at the lowest (highest) beam energies. The conclusions drawn from the comparisons of experimental and simulated IMF sphericities in the ${ }^{129} \mathrm{Xe}+{ }^{139} \mathrm{La}$ reactions, shown in Fig. 22, are similar to those drawn from the comparison of sphericities shown in Fig. 16. The data are between the SB and MF model predictions at the lowest beam energies, and are equal to, or in slight excess of, the MF model predictions at beam energies higher than $\sim 40 \mathrm{MeV} /$ nucleon.

As noted above, the IMF emission patterns in the central ${ }^{12} \mathrm{C}+{ }^{12} \mathrm{C},{ }^{20} \mathrm{Ne}+{ }^{27} \mathrm{Al}$, and ${ }^{40} \mathrm{Ar}+{ }^{45} \mathrm{Sc}$ reactions are far more deformed than that expected from the $\mathrm{SB}$ (or $\mathrm{MF}$ ) model calculations. It is possible to imagine several possible causes for this effect. The first concerns impact parameter fluctuations. In the lightest entrance channels, there is simply not
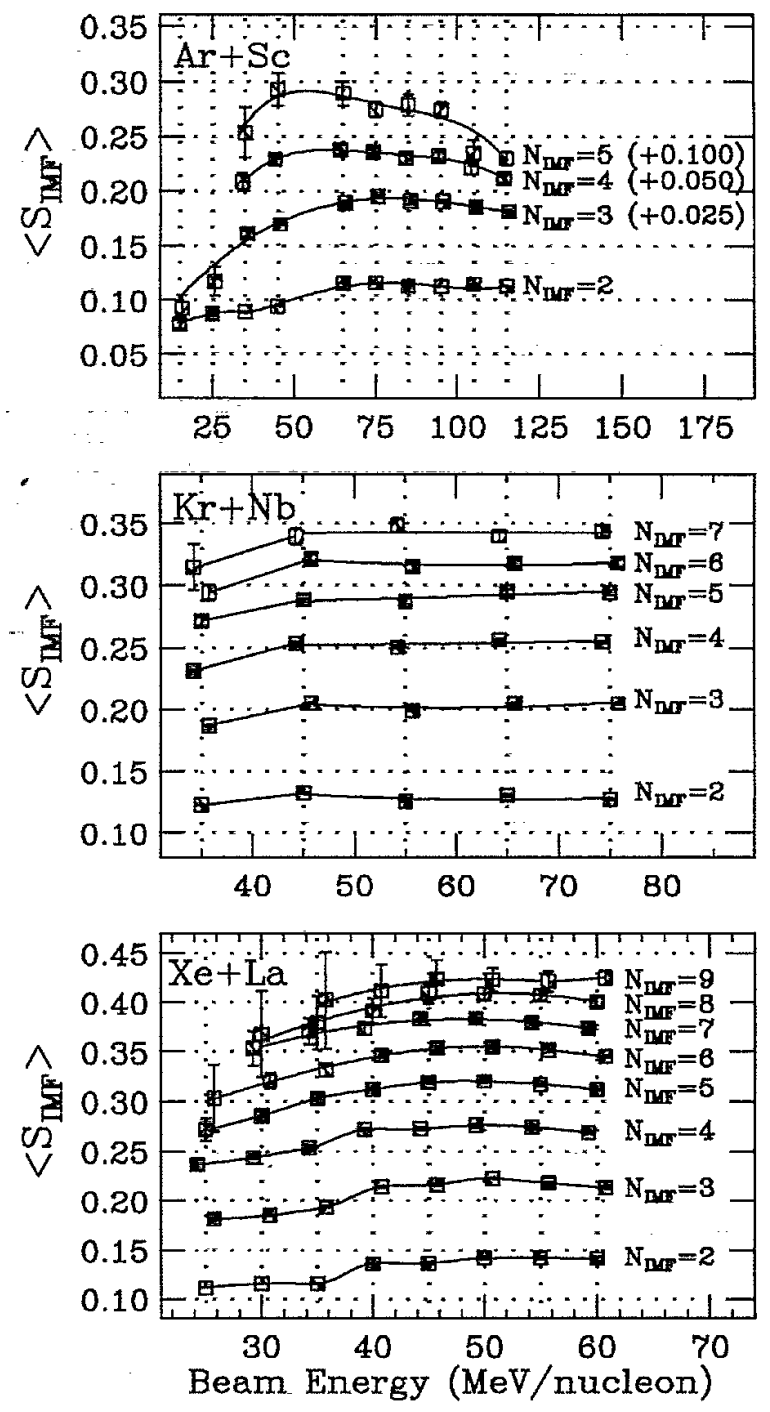

FIG. 20. The average IMF sphericity versus the beam energy for specific IMF multiplicities in the central ${ }^{40} \mathrm{Ar}+{ }^{45} \mathrm{Sc},{ }^{84} \mathrm{Kr}+{ }^{93} \mathrm{Nb}$, and ${ }^{129} \mathrm{Xe}+{ }^{139} \mathrm{La}$ reactions.

much information available upon which to base a centrality cut. An (integer) centrality variable's bin width may be a significant fraction of the maximum value in the spectrum populated in the minimum bias events. As noted in Ref. [17], this leads to relatively larger fluctuations in the impact parameters deduced for each event. If this were the only possibility, a breakdown in the geometrical assumption that the maximal $4-8 \%$ of the minimum bias events corresponds to $\langle b\rangle / b_{\max } \sim 0.2-0.28$ in the light entrance channels would be evident. The second possibility assumes that fluctuations in the initial stages of the reactions increase in importance as the entrance channel mass is decreased. In those collisions for which the equilibration of the excited composite system is particularly incomplete, some knowledge of the initial trajectories of the projectile and target nuclei could be retained by the particles in the final state. Prolate shapes oriented along the beam direction would be expected for such events, in similarity to that expected given significant contaminations to the samples of selected central events from more peripheral collisions. 


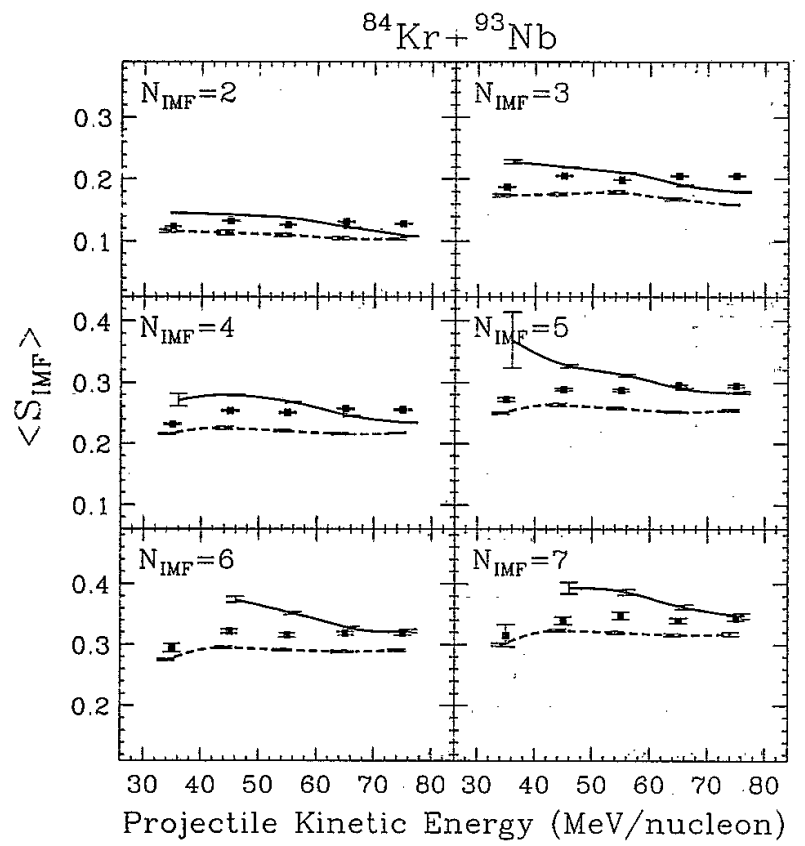

FIG. 21. The average IMF sphericity versus the beam energy for the central ${ }^{84} \mathrm{Kr}+{ }^{93} \mathrm{Nb}$ reactions at specific values of the IMF multiplicity as labeled in each frame. The SB(MF) model results are shown as the dashed(solid) lines. The error bars are statistical, and the abscissa for the model predictions have been shifted by \pm 1 $\mathrm{MeV}$ for clarity.

The third possibility assumes the formation of noncompact geometries other than bubbles, e.g., toroids [28], at freeze-out. In the context of shape analyses, these freeze-out configurations should result in coplanar IMF emission patterns with the IMF flow angles $\theta_{2}=\cos ^{-1}\left(\mathbf{t}_{2} \cdot \hat{\mathbf{z}}\right)$ and $\theta_{3}=\cos ^{-1}\left(\mathbf{t}_{3} \cdot \hat{\mathbf{z}}\right)$ near $90^{\circ}$, where $\hat{\mathbf{z}}$ is the incident beam axis and $t_{2}\left(t_{3}\right)$ is the IMF eigenvector corresponding to the second largest (largest) eigenvalue. The average IMF coplanarity, $\quad C_{\mathrm{IMF}}=(\sqrt{3} / 2)\left(q_{2}-q_{1}\right)$, and flatness, $F_{\mathrm{IMF}}$ $=(\sqrt{3} / 2)\left(t_{2}-t_{1}\right) /\left(\sum_{i=1}^{3} t_{i}\right)$, should exceed that from an isotropic IMF emitter. One would furthermore expect that $C_{\mathrm{IMF}}$ and $F_{\mathrm{IMF}}$ would increase with both the IMF flow angle $\theta_{3}$ and the sum $\theta_{3}+\theta_{2}$ (for specific values of $N_{\mathrm{IMF}}$ ).

Other aspects of the average shapes of the IMF's in the central events were studied to investigate these possibilities. This study implies that the IMF shapes in the selected ${ }^{40} \mathrm{Ar}+{ }^{45} \mathrm{Sc}$ events (and in the lighter entrance channels) are manifestly prolate. For all values of $N_{\mathrm{IMF}}$, the central event averaged probability that $t_{3}-t_{2}>t_{2}-t_{1}$, corresponding to prolate IMF emission patterns, increases significantly for decreasing entrance channel mass. These probabilities are shown in Fig. 23. The $\mathrm{CM}$ frame ratios $\langle E\rangle / 1.5\left\langle E_{T}\right\rangle$ are shown versus the particle charge in Fig. 24, where $E\left(E_{T}\right)$ is the total (transverse) kinetic energy. A prolate(oblate) pattern with the symmetry axis near the beam axis will result in $\left.\langle E\rangle / 1.5\left\langle E_{T}\right\rangle\right\rangle(<) 1$, while a spherical pattern will result in values near unity. The experimental values of this ratio in the selected events increase with decreasing entrance channel mass.

These observations point to an increase in the probability that the IMF emission patterns are elongated along the beam

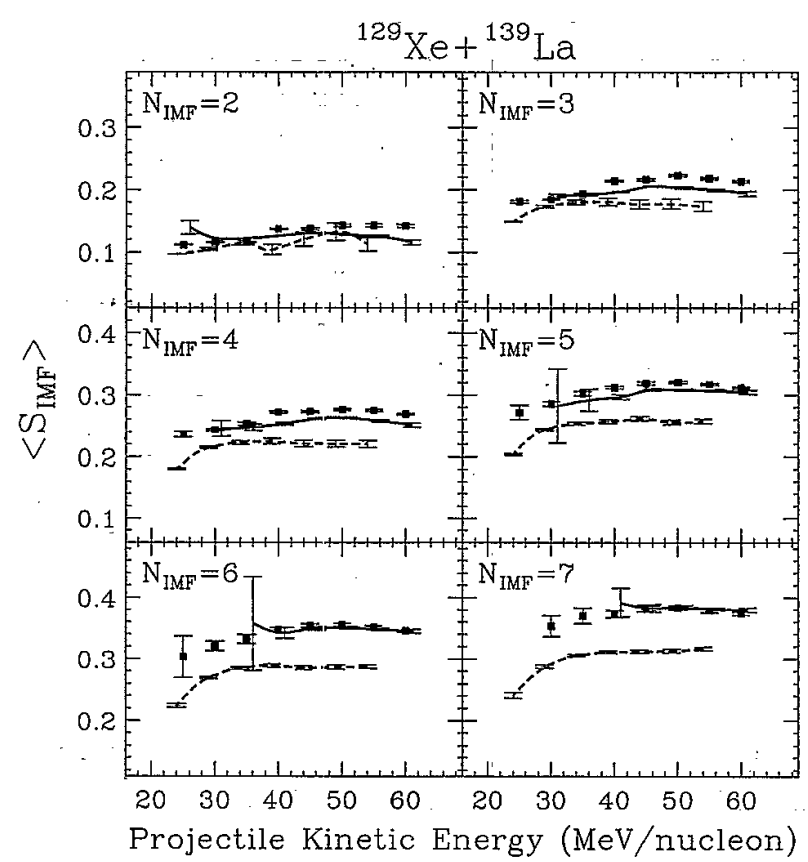

FIG. 22. The same as Fig. 21, but for the central ${ }^{129} \mathrm{Xe}+{ }^{139} \mathrm{La}$ reactions.

direction as the entrance channel mass is decreased, despite the restrictive central event selection described in Sec. I. This is taken to imply an increase in the importance of impact parameter fluctuations, and/or an increase in the fluctuations in the initial stages of the reactions, as the entrance channel mass is decreased. This observation makes remote the possibility that the IMF emission in these data proceeds via a noncompact geometry that is oblate and expanding in the $X-Y$ plane, as suggested by various transport calculations [28-30].

Further evidence that the observed IMF's do not freeze out from a toroidal (or disk) shaped system is provided by Figs. 18 and 19. Such geometries have been seen in various BUU calculations [28-30] of central collisions in entrance channels that are the same or similar to the ${ }^{84} \mathrm{Kr}+{ }^{93} \mathrm{Nb}$ and ${ }^{129} \mathrm{Xe}+{ }^{139} \mathrm{La}$ reactions studied here. However, the IMF sphericities observed in these entrance channels are consistent with the predictions of the present MF model calculations, which assume an expanded, but spherical, freeze-out volume.

It has been noted [30] that an observed absence of such planar configurations and the observations of spherical IMF emission is a statement that the nuclear equation of state is "soft" ( $\left.K_{\infty} \sim 215 \mathrm{MeV}\right)$. We note, however, that, if the IMF's that freeze out of such a torus or disk expand (in the $X-Y$ plane) with a transverse energy less than a few $\mathrm{MeV} /$ nucleon for beam energies above $\sim 50 \mathrm{MeV} /$ nucleon, then they will be more forward of the acceptance of the $4 \pi$ Array in its present configuration.

\section{SUMMARY AND CONCLUSIONS}

This work concentrated on a particular projection, called the sphericity, of the shapes in momentum space of events from central heavy-ion reactions. A data set that is compre- 


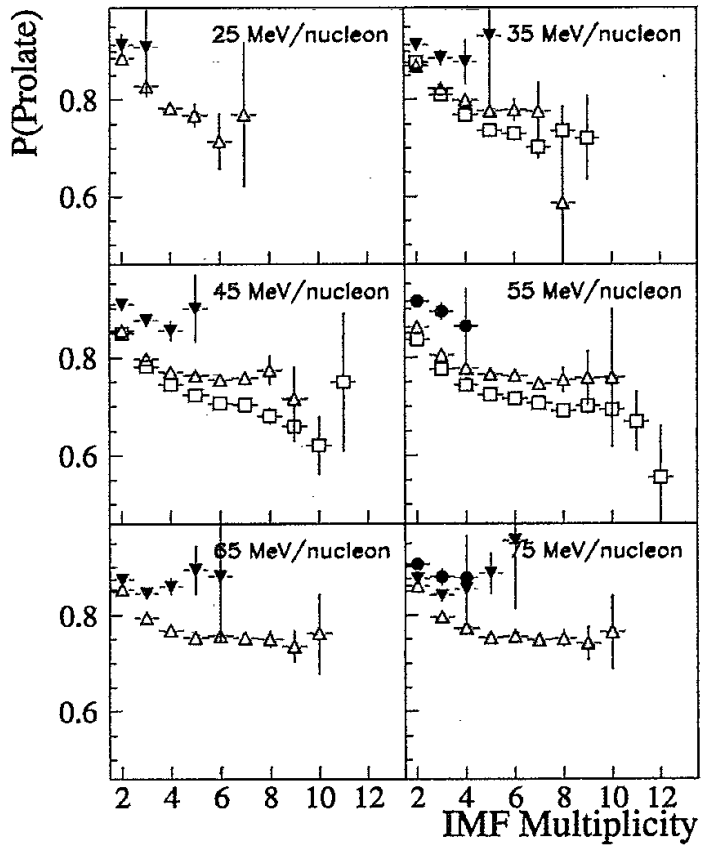

FIG. 23. The probability for prolate IMF emission versus the IMF multiplicity for the central ${ }^{20} \mathrm{Ne}+{ }^{27} \mathrm{Al}$ (solid circles), ${ }^{40} \mathrm{Ar}+{ }^{45} \mathrm{Sc}$ (solid triangles), ${ }^{84} \mathrm{Kr}+{ }^{93} \mathrm{Nb}$ (open triangles), and ${ }^{129} \mathrm{Xe}+{ }^{139} \mathrm{La}$ reactions (open squares) at the beam energies listed in each frame. The specific definition of this probability is described in the text.

hensive in both the entrance channel mass and the beam energy was studied.

The relative efficiencies of many different methods for the selection of the small impact parameter events were investigated. The particular choice of the global observable upon

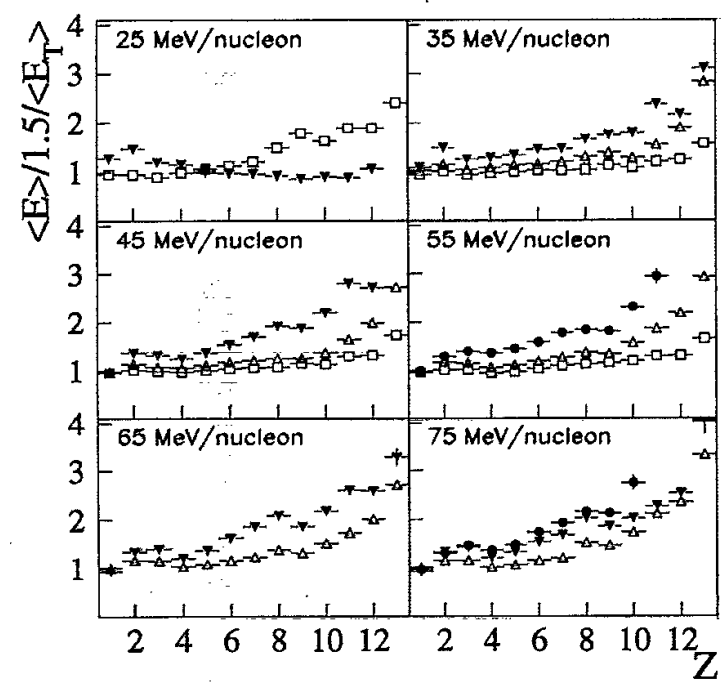

FIG. 24. The ratio of the average kinetic energy and one and one half times the average transverse kinetic energy of the particles in the selected central events, versus the particle charge. The results for the ${ }^{20} \mathrm{Ne}+{ }^{27} \mathrm{Al}$ (solid circles), ${ }^{40} \mathrm{Ar}+{ }^{45} \mathrm{Sc}$ (solid triangles), ${ }^{84}$ $\mathrm{Kr}+{ }^{93} \mathrm{Nb}$ (open triangles), and ${ }^{129} \mathrm{Xe}+{ }^{139} \mathrm{La}$ reactions (open squares) are shown.

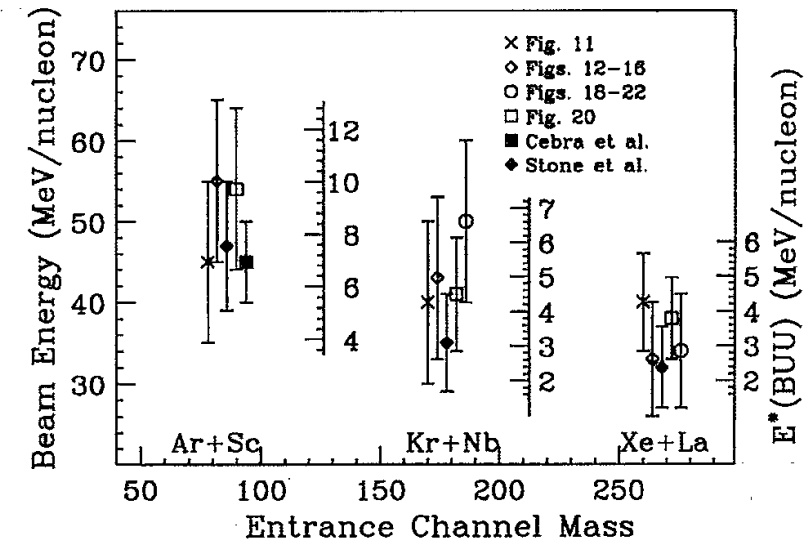

FIG. 25. A summary of the results of the present shape analyses indicating transitions in various characteristics of the predominant reaction mechanisms for the systems formed in the central collisions in the different entrance channels. Predictions of the BUU calculations for the approximate excitation energies reached in the selected events are given for each entrance channel on the right side axes.

which centrality cuts are placed was shown to significantly affect the sphericities of the events that are selected. The most efficient centrality variables were assumed to result in the most spherical events, following the removal of the finite multiplicity distortions $[10,11]$ to the average sphericities. Two new methods were described that allow the removal, on the average, of these distortions. These allowed the ranking of the relative efficiencies of the different centrality variables for the selection of small impact parameters to be experimentally accessible. Transitions in the variables that best select the most central events were observed in the three heaviest entrance channels, which is indicative of significant changes in the topology of the central events. This was presumed to be indicative of changes in the predominant decay mechanism, and was further investigated in the remainder of this paper. Autocorrelations [7] between the average sphericity and the various centrality variables were also investigated, and were shown to be negligible for the $\sim 10 \%$ cuts [19] used in the present analyses.

The average sphericities of the central events, and of a particular subset of these events (the IMF's), were compared to those predicted by a variety of filtered SB and MF model codes. Unlike the studies of relative sphericities described in Sec. I, these comparisons allowed tests of the absolute scale of average sphericities from the data and the models. Transitions from SB disassembly to MF were observed on the basis of these comparisons. However, the IMF emission in the light entrance channels is manifestly prolate along the beam direction, despite the restrictive selection of central events that was performed. In the heavier entrance channels and for beam energies above $\sim 40 \mathrm{MeV} /$ nucleon, the IMF emission is consistent with the predictions of the MF models, which assume an expanded and spherical freeze-out configuration. This casts doubt on the transport model predictions of freeze-out configurations that are tori or disks and expanding in the $X-Y$ plane.

There are no indications of $\mathrm{SB}$ to $\mathrm{MF}$ transitions in the available central ${ }^{12} \mathrm{C}+{ }^{12} \mathrm{C}$ and ${ }^{20} \mathrm{Ne}+{ }^{27} \mathrm{~A} 1$ reactions 
( $E_{\text {proj }} \geqslant 55 \mathrm{MeV} /$ mucleon). The qualitatively located beam energies at which the various analyses described in this paper indicated $\mathrm{SB}$ to $\mathrm{MF}$ transitions in the central ${ }^{40} \mathrm{Ar}+{ }^{45} \mathrm{Sc}$, ${ }^{84} \mathrm{Kr}+{ }^{93} \mathrm{Nb}$, and ${ }^{129} \mathrm{Xe}+{ }^{139} \mathrm{La}$ reactions are depicted in Fig. 25. Also included in this figure is the transitional beam energy observed by Cebra et al. for central ${ }^{40} \mathrm{Ar}+{ }^{51} \mathrm{~V}$ reactions [26], and the transitional beam energies observed in a charge correlation analysis of the present data [31]. In this analysis, a clear transition in the relative sizes of the three largest fragments in the central events is observed at beam energies of $47 \pm 10,35 \pm 10$, and $32 \pm 5 \mathrm{MeV} /$ nucleon in the ${ }^{40} \mathrm{Ar}+{ }^{45} \mathrm{Sc},{ }^{84} \mathrm{Kr}+{ }^{93} \mathrm{Nb}$, and ${ }^{129} \mathrm{Xe}+{ }^{139} \mathrm{La}$ entrance channels, respectively.

The various analyses indicate similar transitional beam energies for each entrance channel. The transitional beam energies observed for the central ${ }^{40} \mathrm{Ar}+{ }^{45} \mathrm{Sc}$ reactions are significantly larger than those observed for the central ${ }^{84} \mathrm{Kr}+{ }^{93} \mathrm{Nb}$ and ${ }^{129} \mathrm{Xe}+{ }^{139} \mathrm{La}$ reactions.

The most obvious possibilities for this trend concern the open questions raised in Secs. II and III. These involve an increasing importance of fluctuations in the impact parameter, or in the degree of equilibrium of the excited systems formed at one impact parameter, for decreasing entrance channel mass. Two other possibilities are noted, which should not, however, be considered until the two above have been fully explored. The third assumes an increase in the importance of quantum mechanical finite-size effects for the excited systems formed in increasingly lighter entrance channels. The fourth involves the fact that increasingly heavier entrance channels result in excited systems that are increasingly more proton rich. The model calculations described in Sec. II predict a significant decrease in the transitional beam energy with an increase in the Coulomb energy of a nuclear system of fixed total mass. Experiments in several entrance channels of constant total mass but differing charge/mass ratios would be needed to verify this prediction.

\section{ACKINOWLEDGMENTS}

We gratefully acknowledge helpful discussions with $\mathrm{P}$. Danielewicz and L. Phair. This work was supported by the U.S. Department of Energy under the Grant No. DE-FG0393ER40772, and the U.S. National Science Foundation under Grants No. PHY 89-13815, No. PHY 92-14992, and No. PHY 91-20728.
[1] H. Fuchs and K. Möhring, Rep. Prog. Phys. 57, 231 (1994), and references therein; L. G. Moretto and G. J. Wozniak, Annu. Rev. Nucl. Part. Sci. 43, 379 (1993), and references therein; D. H. E. Gross, Rep. Prog. Phys. 53, 605 (1990), and references therein; E. Saraud, Ch. Grégoire and B. Tamain, Prog. Nucl. Part. Sci. 23, 357 (1989), and references therein.

[2] J. P. Bondorf, R. Donangelo, I. N. Mushustin, C. J. Pethick, H. Schulz, and K. Sneppen, Nucl. Phys. A443, 321 (1985); J. P. Bondorf, R. Donangelo, I. N. Mushustin, and H. Schulz, ibid. A444, 460 (1985); H. W. Barz, J. P. Bondorf, R. Donangelo, I. N. Mushustin, and H. Schulz, ibid. A448, 753 (1986).

[3] A. S. Botvina, I. S. Пjinov, I. N. Mushustin, J. P. Bondorf, R. Donangelo, and K. Sneppen, Nucl. Phys. A475, 663 (1987).

[4] W. Bauer, Phys. Rev. C 38, 1297 (1988).

[5] G. D. Westfall, J. E. Yurkon, J. Van Der Plicht, Z. M. Koenig, B. V. Jacak, R. Fox, G. M. Crawley, M. R. Maier, B. E. Hasselquist, R. S. Tickle, and D. Horn, Nucl. Instrum. Methods Phys. Res. Sect. A 238, 347 (1985); M. Maier, M. Robertson, A. Vander Molen, and G. D. Westfall, ibid. 337, 619 (1994); A. M. Vander Molen, R. Au, R. Fox, and S. Hannuschke, IEEE Trans. Nucl. Sci. 41, 80 (1994).

[6] G. D. Westfall, W. Bauer, D. Craig, M. Cronqvist, E. Gualtieri, S. Hannuschke, D. Klakow, T. Li, T. Reposeur, A. M. Vander Molen, W. K. Wilson, J. S. Winfield, J. Yee, S. J. Yennello, R. A. Lacey, A. Elmaani, J. Lauret, A. Nadasen, and E. Norbeck, Phys. Rev. Lett. 71, 1986 (1993); E. Bauge, A. Elmaani, R. A. Lacey, J. Lauret, N. N. Ajitanand, D. Craig, M. Cronqvist, E. Gualtieri, S. Hannuschke, T. Li, W. J. Llope, T. Reposeur, A. M. Vander Molen, G. D. Westfall, J. S. Winfield, J. Yee, S. J. Yennello, A. Nadasen, and E. Norbeck, ibid. 70, 3705 (1993); T. Li, W. Bauer, D. Craig, M. Cronqvist, E. Gualtieri, S. Hannuschke, R. A. Lacey, W. J. Llope, T. Reposeur, A. M. Vander Molen, G. D. Westfall, W. K. Wilson, J. S. Winfield, J. Yee, S.
J. Yennello, A. Nadasen, R. S. Tickle, E. Norbeck, ibid. 70, 1924 (1993); R. A. Lacey, A. Elmaani, J. Lauret, T. Li, W. Bauer, D. Craig, M. Cronqvist, E. Gualtieri, S. Hannuschke, T. Reposeur, A. M. Vander Molen, G. D. Westfall, W. K. Wilson, J. S. Winfield, J. Yee, S. J. Yennello, A. Nadasen, R. S. Tickle, and E. Norbeck, ibid. 70, 1224 (1993).

[7] W. J. Llope, J. A. Conrad, C. M. Mader, G. Peilert, W. Bauer, D. Craig, E. Gualtieri, S. Hannuschke, R. A. Lacey, J. Lauret, T. Li, A. Nadasen, E. Norbeck, R. Pak, N. Stone, A. M. Vander Molen, G. D. Westfall, J. Yee, and S. J. Yennello, Phys. Rev. C 51, 1325 (1995).

[8] M. Gyulassy, K. A. Frankel, and H. Stöcker, Phys. Lett. 110B, 185 (1982).

[9] G. Fái and J. Randrup, Nucl. Phys. A381, 557 (1982); A404, 551 (1983).

[10] P. Danielewicz and M. Gyulassy, Phys. Lett. 129B, 283 (1983).

[11] J. P. Bondorf, C. H. Dasso, R. Donangelo, and G. Pollarolo, Phys. Lett. B 240, 28 (1990).

[12] H. Stöcker, G. Buchwald, L. P. Csernai, G. Graebner, J. A. Maruhn, and W. Greiner, Nucl. Phys. A387, 205c (1982).

[13] J. A. López and J. Randrup, Nucl. Phys. A491, 477 (1989).

[14] C. Cavata, M. Demoulins, J. Gosset, M. C. Lemaire, D. L'Hôte, J. Poitou, and O. Valette, Phys. Rev. C 24, 1760 (1990).

[15] M. B. Tsang, Y. D. Kim, N. Carlin, Z. Chen, R. Fox, C. K. Gelbke, W. G. Gong, W. G. Lynch, T. Murakami, T. K. Nayak, R. M. Ronningen, H. M. Xu, F. Zhu, L. Sobotka, D. Stracener, D. G. Sarantites, Z. Majka, V. Abenante, and H. Griffin, Phys. Lett. B 220, 492 (19:39).

[16] C. A. Ogilvie, D. A. Cebra, J. Clayton, S. Howden, J. Karn, A. Vander Molen, G. D. Westfall, W. K. Wilson, and J. S. Winfield, Phys. Rev. C 40, 654 (1989).

[17] L. Phair, D. R. Bowman, C. K. Gelbke, W. G. Gong, Y. D. 
Kim, M. A. Lisa, W. G. Lynch, G. F. Peaslee, R. T. de Souza, M. B. Tsang, and F. Zhu, Nucl. Phys. A548, 489 (1992).

[18] L. Phair, D. R. Bowman, N. Carlin, C. K. Gelbke, W. G. Gong, Y. D. Kim, M. A. Lisa, W. G. Lynch, G. F. Peaslee, R. T. de Souza, M. B. Tsang, C. Williams, F. Zhu, N. Colonna, K. Hanold, M. C. McMahon, and G. J. Wozniak, Nucl. Phys. A564, 453 (1993).

[19] It is not always possible to select a specific fraction of the impact parameter inclusive events, as often the case for centrality variables that are integer quantities. The threshold chosen for a particular centrality variable was the lowest possible bin for which $\leqslant 10 \%$ of the minimum bias events are in and above this bin. Generally, $7-10 \%$ of the events are selected by these cuts.

[20] C. M. Mader, Ph.D. thesis, Michigan State University, 1993 (unpublished); W. Bauer, G. F. Bertsch, W. Cassing, and U. Mosel, Phys. Rev. C 34, 2127 (1986); W. Bauer, Phys. Rev. Lett. 61, 2534 (1988).

[21] J. Aichelin and H. Stöcker, Phys. Lett. B 176, 14 (1986); J. Aichelin, G. Peilert, A. Bohnet, A. Rosenhauer, H. Stöcker, and W. Greiner, Phys. Rev. C 37, 2451 (1988); G. Peilert, J. Konopka, H. Stöcker, W. Greiner, M. Blann, and M. G. Mustafa, ibid. 46, 1457 (1992).

[22] D. R. Bowman, C. M. Mader, G. F. Peaslee, W. Bauer, N. Carlin, R. T. de Souza, C. K. Gelbke, W. G. Gong, Y. D. Kim, M. A. Lisa, W. G. Lynch, L. Phair, M. B. Tsang, C. Williams, N. Colonna, K. Hanold, M. A. McMahan, G. J. Wozniak, L. G. Moretto, and W. A. Friedman, Phys. Rev. C 46, 1834 (1992).

[23] A. R. DeAngelis and D. H. E. Gross (private communication);
D. H. E. Gross, Prog. Part. Nucl. Phys. 30, 155 (1993), and references therein; X. -Z. Zhang, D. H. E. Gross, S. -Y. Xu, and Y. -M. Zheng, Nucl. Phys. A461, 641 (1987); A461, 668 (1987).

[24] H. W. Barz, D. A. Cebra, H. Schulz, and G. D. Westfall, Phys. Lett. B 267, 317 (1991).

[25] R. J. Charity, M. A. McMahan, G. J. Wozniak, R. J. McDonald, L. G. Moretto, D. G. Sarantites, L. G. Sobotka, G. Guarino, A. Pantaleo, L. Fiore, A. Gobbi, and K. D. Hildenbrand, Nucl. Phys. A483, 371 (1988).

[26] D. A. Cebra, S. Howden, J. Karn, A. Nadasen, C. A. Ogilvie, A. Vander Molen, G. D. Westfall, W. K. Wilson, and J. S. Winfield, Phys. Rev. Lett. 64, 2246 (1990).

[27] G. Casini et al., Phys. Rev. Lett. 71, 2567 (1993); H. Delagrange, C. Grégoire, F. Scheuter, and Y. Abe, Z. Phys. A 323, 437 (1986).

[28] W. Bauer, G. F. Bertsch, and H. Schulz, Phys. Rev. Lett. 69, 1888 (1992); L. G. Moretto, K. Tsuo, N. Colonna, and G. J. Wozniak, ibid. 69, 1884 (1992).

[29] L. Phair, W. Bauer, and C. K. Gelbke, Phys. Lett. B 314, 271 (1994); T. Glasmacher, C. K. Gelbke, and S. Pratt, ibid. 314, 265 (1993); D. H. E. Gross, B. -A. Li, and A. R. DeAngelis, Ann. Phys. (Leipzig) 1, 467 (1992); S. R. Souza and C. Ngô, Phys. Rev. C (submitted).

[30] H. M. Xu, J. B. Natowitz, C. A. Gagliardi, R. E. Tribble, C. Y. Wong, and W. G. Lynch, Phys. Rev. C 48, 933 (1993).

[31] N. Stone, W. J. Llope, and G. D. Westfall, Phys. Rev. C 51, 3157 (1995). 